ARTICLE

\title{
Phosphoglycolate phosphatase homologs act as glycerol-3-phosphate phosphatase to control stress and healthspan in C. elegans
}

Elite Possik (iD 1,2, Clémence Schmitt (10 1,2, Anfal Al-Mass (i] 1,2, Ying Bai 1,2, Laurence Côté (iD 1,2, Johanne Morin ,2,

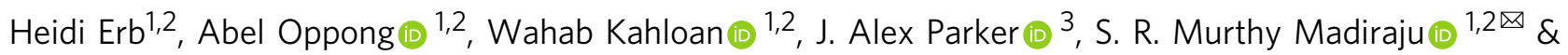
Marc Prentki ${ }^{1,2}$ 厛

Metabolic stress due to nutrient excess and lipid accumulation is at the root of many ageassociated disorders and the identification of therapeutic targets that mimic the beneficial effects of calorie restriction has clinical importance. Here, using $C$. elegans as a model organism, we study the roles of a recently discovered enzyme at the heart of metabolism in mammalian cells, glycerol-3-phosphate phosphatase (G3PP) (gene name Pgp) that hydrolyzes glucose-derived glycerol-3-phosphate to glycerol. We identify three Pgp homologues in C. elegans ( $p g p h)$ and demonstrate in vivo that their protein products have G3PP activity, essential for glycerol synthesis. We demonstrate that PGPH/G3PP regulates the adaptation to various stresses, in particular hyperosmolarity and glucotoxicity. Enhanced G3PP activity reduces fat accumulation, promotes healthy aging and acts as a calorie restriction mimetic at normal food intake without altering fertility. Thus, PGP/G3PP can be considered as a target for age-related metabolic disorders.

\footnotetext{
${ }^{1}$ Department of Nutrition, Université de Montréal, Montreal Diabetes Research Center, CRCHUM, Montréal, Canada. ${ }^{2}$ Department of Biochemistry and Molecular Medicine, Montreal Diabetes Research Center, CRCHUM, Montréal, Canada. ${ }^{3}$ Department of Neurosciences, CRCHUM, Montréal, Canada.

凶email: s.r.murthy.madiraju.chum@ssss.gouv.qc.ca; marc.prentki@umontreal.ca
} 
G lycerolipid/free fatty acid (GL/FFA) cycle links lipid and glucose metabolism and its dysregulation is associated with metabolic and age-related diseases ${ }^{1,2}$. This cycle, consisting of its lipogenesis and lipolysis arms, generates signaling molecules and promotes thermogenesis. During lipogenesis, glucose-derived glycerol-3-phosphate (Gro3P) is sequentially esterified with fatty acyl-CoAs to form triglycerides (TG). Upon limited nutrient supply, stored TG is hydrolyzed to glycerol and FFA $^{1,2}$.

In biochemistry textbooks, lipolysis in mammals was believed to be the only source of glycerol, since they were thought to lack enzyme(s) converting Gro3P to glycerol. We have discovered a Gro3P phosphatase (G3PP) enzyme capable of hydrolyzing Gro3P to glycerol in mammalian cells ${ }^{3}$. Gro3P is a central metabolite at the crossroads of fat and carbohydrate metabolism. G3PP suppression in hepatocytes and pancreatic $\beta$-cells increased lipid synthesis, reduced FFA oxidation and oxygen consumption, and lowered ATP production. Our findings implicated G3PP in the control of glycolysis, gluconeogenesis, cellular redox, and energy production when glucose is elevated ${ }^{3}$. However, the pathophysiological roles of G3PP in vivo are largely unknown.

Gro3P hydrolysis is a newly identified function of the previously described enzyme phosphoglycolate phosphatase (gene: $P g p)^{4}$ that belongs to the superfamily of haloacid dehalogenases (HAD)-like hydrolases. PGP also shows phosphatase activity in vitro towards several substrates besides Gro3 $\mathrm{P}^{3,5,6}$, including 2-phosphoglycolate (2-PG) produced during DNA repair ${ }^{7}$, glycolysis-derived toxic side products such as 2-phospholactate and 4-phosphoerythronate ${ }^{8}$, and very low activity towards phosphotyrosine residues and nucleotides ${ }^{3,9}$. However, the physiological relevance of these activities remains to be defined in vivo, as the cellular concentrations of these metabolites are very low under normal culture conditions ${ }^{5}$.

Gro3P is a key intermediate of glycolysis, glycerolipid biosynthesis, gluconeogenesis, and the Gro3P redox shuttle. In conditions of excess glucose, Gro3P is redirected towards lipogenesis and the Gro3P shuttle, leading to fat deposition and metabolic stress/ ROS production, respectively, contributing to tissue damage and premature aging ${ }^{10-14}$. In contrast, dietary restriction is associated with reduced metabolism and it delays disease onset and extends lifespan in invertebrate ${ }^{15-22}$, vertebrate organisms $s^{23-25}$, and humans ${ }^{26}$. However, outstanding drawbacks of calorie restriction are that a substantial reduction in food intake is not applicable to humans and causes a major reduction in fertility. Thus, there is an outstanding interest in identifying pathways that could mimic calorie restriction without its associated caveats. The fact that G3PP hydrolyzes glucose-derived Gro3P to glycerol, a much less toxic molecule when produced in excess ${ }^{27}$, implies that this pathway might protect organisms from glucotoxicity and nutri-stress ${ }^{28}$. In addition, removal of glucose carbons as glycerol, that exit the cell through aquaglyceroporins, could mimic calorie restriction by reducing the availability of glucose for oxidation and lipogenesis. However, these candidate beneficial roles of G3PP have not been investigated in vivo.

Other than its metabolic functions, glycerol is an essential protective organic osmolyte produced to adapt high salinity environments ${ }^{29,30}$. In Caenorhabditis elegans (C. elegans), exposure to hyperosmotic stress causes a rapid glycerol accumulation and is accompanied by the upregulation of mRNA levels of glycerol-3-phosphate dehydrogenase enzymes, $g p d h-1$ and $g p d h-2$ and glycogenolysis ${ }^{31-35}$. The roles of $g p d h-1$ and $g p d h-2$ enzymes have been examined in regard with hyperosmotic stress in $C$. elegans $^{31,32,36,37}$. However, how Gro3P leads to glycerol synthesis downstream salt-driven glycogenolysis, and whether glycerol could be rapidly formed by an overlooked G3PP that mediates the hydrolysis of Gro3P in C. elegans, is unknown.
To identify G3PP functions in vivo, we used C. elegans as a model system and studied many complex processes, particularly the response to various stresses and aging. Here, we show that C. elegans harbors three homologs of mammalian G3PP that we named phosphoglycolate phosphatase homologs (now listed as PGPH; WormBase). We demonstrate that the worm PGPH acts as a Gro3P phosphatase in vivo and that G3PP is involved in the response to various stresses, including excess glucose and hyperosmotic stress, as well as healthy aging. Also, we show that enhanced PGPH/G3PP activity reduces fat accumulation and in part mimics the beneficial effects of dietary restriction and promotes healthy aging, particularly in conditions of excess glucose, without adversely affecting the apparent food intake (pharyngeal pumping) or reproduction.

\section{Results}

The worm genome harbors three homologs of mammalian PGP/G3PP with glycerol-3-phosphate phosphatase function. Using blast search, we found three homologs of G3PP in the $C$. elegans genome, K09H11.7, F44E7.2, and C53A3.2 that we named pgph-1, pgph-2, and pgph-3, respectively, in accordance with the WORMBASE guidelines (Fig.1a). The three isozymes present a high level of protein sequence identity to human PGP/G3PP, with highly conserved catalytic motifs (Fig. 1a). Transcriptional reporters of $p g p h-2$ and $p g p h-3$ demonstrate ubiquitous expression of these genes with minor differences, implying possible differential tissue-specific roles (Supplementary Fig. 1a). The expression of pgph-1 was not studied in the present work due to the unavailability of $p g p h-1$ single mutant animals and our data revealed a minor role of this enzyme in the various studied biological functions (see below).

In order to examine the roles of the PGPH isoenzymes and to avoid compensatory effects, we first generated triple ( $p g p h-1$; pgph-2; pgph-3) deletion mutants using CRISPR-CAS-9 technology, that we refer to as triple pgph mutant animals for simplicity throughout the text. Triple pgph mutant animals did not show phenotypic defects in pharyngeal pumping, egg laying, brood size, or swimming behaviors (Supplementary Fig. 1b-e). PGPH isozymes have G3PP activity in vivo as markedly decreased glycerol levels were found in triple pgph mutant animals (Fig. 1b). Accordingly, Gro3P and dihydroxyacetone-phosphate (DHAP) levels were significantly increased in these mutants (Fig. 1c). No significant changes were seen in other glycolytic metabolites as well as Krebs cycle intermediates and adenine and pyrimidine nucleotides (Fig. 1c). Also, levels of 2-phosphoglycolate (2-PG), a metabolite linked to DNA repair and a substrate with which G3PP/PGP shows high activity in vitro, were unchanged suggesting that PGPH functions mostly as a G3PP in the worm under normal in vivo conditions (Fig. 1c). PGPH/G3PP deletion is anticipated to increase lipogenesis and fat deposition. Accordingly, triple pgph mutant animals showed increased oil red $\mathrm{O}$ staining distributed throughout the body, indicating elevated fat deposition (Fig. 1d-f). Overall, these results establish that PGPH isoenzymes in the worm act as Gro3P phosphatase in vivo under normal physiological conditions.

Enhanced lipogenesis mediates fat accumulation in triple $p g p h$ mutant animals. The balance between lipogenesis and lipolysis is critical for metabolic homeostasis. To determine whether lipogenesis is driving fat accumulation in triple pgph mutant animals, we inhibited lipogenesis by RNAi-knockdown of fat synthase-1 (fasn-1) and the desaturase (fat-7), two key genes essential for fat synthesis in C. elegans (Fig. 2a). Importantly, knockdown of fasn1 completely suppressed the increased fat accumulation phenotype observed in triple pgph mutant animals back to Ev levels 
a

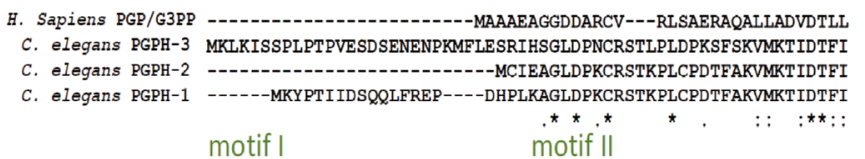

H. Sapiens PGP/G3PP FDCDGVLWRGETAVPGAPEALRALRARGKRLGFITNNSSKTRAAYAEKLRRLGFGGPAGP

c. elegans PGPH-3 FDADGVLWLGESVMPGSPRLIDYLVKHNKQI IVLTNNATKSRAVYAKKLAKLGYNSSKMN

c. elegans PGPH-2 FDADGVLWLGESVMPGSPRLIDYIVKHNKQIIVLTNNATKSRAVYAKKLAKLGYNSSKMMI

c. elegans PGPH-1 FDADGVLWLGESVMPGSPRLIDYLVKHNRQIIVLTNNATRSRAVYAKKLAKLGYNSSKMN

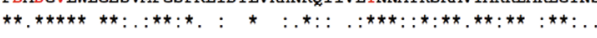

H. Sapiens PGP/G3PP GASLEVFGTAYCTALYLRQRLAGAPAPKAYVLGSPALAAELEAVGVASVGVGPEPLQGEG

C. elegans PGPH-3 KNNLVNPAAV----VADTLHRAGLDGKRVYLIGEQGLRDEMDELGIEYFGHGPEKKQDEA

C. elegans PGPH-2 KNNLVNPAAV----VADTLHRAGLDGKRVYLIGEQGLRDEMDELGIEYFGHGPEKKQDER

c. elegans PGPH-1 KNNLVNPAAV----VADTLHRAGLDGKRVYLIGEQGLRDEMDELGIEYFGHGPEKKQDEA

H. Sapiens PGP/G3PP PG--DW-LHAPLEPDVRAVVVGFDPHFSYMKLTKALRYLQQPGCLLVGTNMDNRLPLENG

c. elegans PGPH-3 DGSGAFMYDIKLEENVGAVVVGYEKHFDYVKMMKASNYLREEGVLFVATNEDETCPGPNP

c. elegans PGPH-2 DGSGAFMYDIKLEENVGAVVVGYEKHFDYVKMMIKASNYLREEGVLFVATNEDETCPGPND

c. elegans PGPH-1 DGSGAFMYDIKLEENVGAVVVGYEKHFDYIKMMIKASNYLREEGVLFVATNEDETCPGPNP

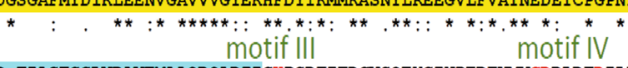

H. Sapiens PGP/G3PP R-FIAGTGCLVRAVEMAAQRQADIIGKPSRFIFDCVSQEYGINPERTVMVGDRLDTDILL C. elegans PGPH-3 EVVIPDAGPIVAAIKCASGRDPLTVGKPCTPAFNYIKRKWNINPSRTMMIGDRTNTDVKF c. elegans PGPH-2 EVVI PDAGPIVAAIKCASGRDPLTVGKPCTPAFNYIKRKWNINPSRTMMIGDRTNTDVKF c. elegans PGPH-1 EVVIPDAGPIVAAIKCASGRDPLTVGKPCTPAFNYIKRKWNINPSRTMMIGDRTNTDVKF

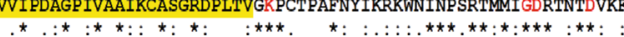

H. Sapiens PGP/G3PP GATCGLKTILTLTGVSTLGDVKNNQESDCVSKKKMVPDFYVDSIADLLPALQG---

c. elegans PGPH-3 GRDHGMKTLIVLSGCHQIEDIIENQMN---ERDDMVPDYVAPYLGALVPESNQIYY

c. elegans PGPH-2 GRDHGMKTLLVLSGCHOIEDIIENOMN---ERDDMVPDYVAPCLGALVPESNQIYY

c. elegans PGPH-1 GRDHGMKTLIVLSGCHQIEDIIENQMN---ERDDMVPDYVAPCLGALVPERI----

GRDHGMKTLIVLSGCHQIEDIIENQMN---ERDDMVPDYVAPCLGALVPERI

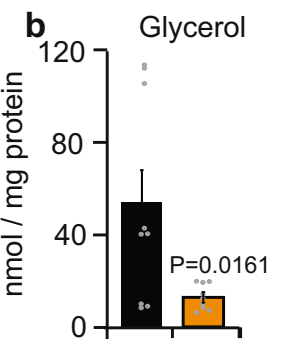

wt

pgph-1; pgph-2; pgph-3
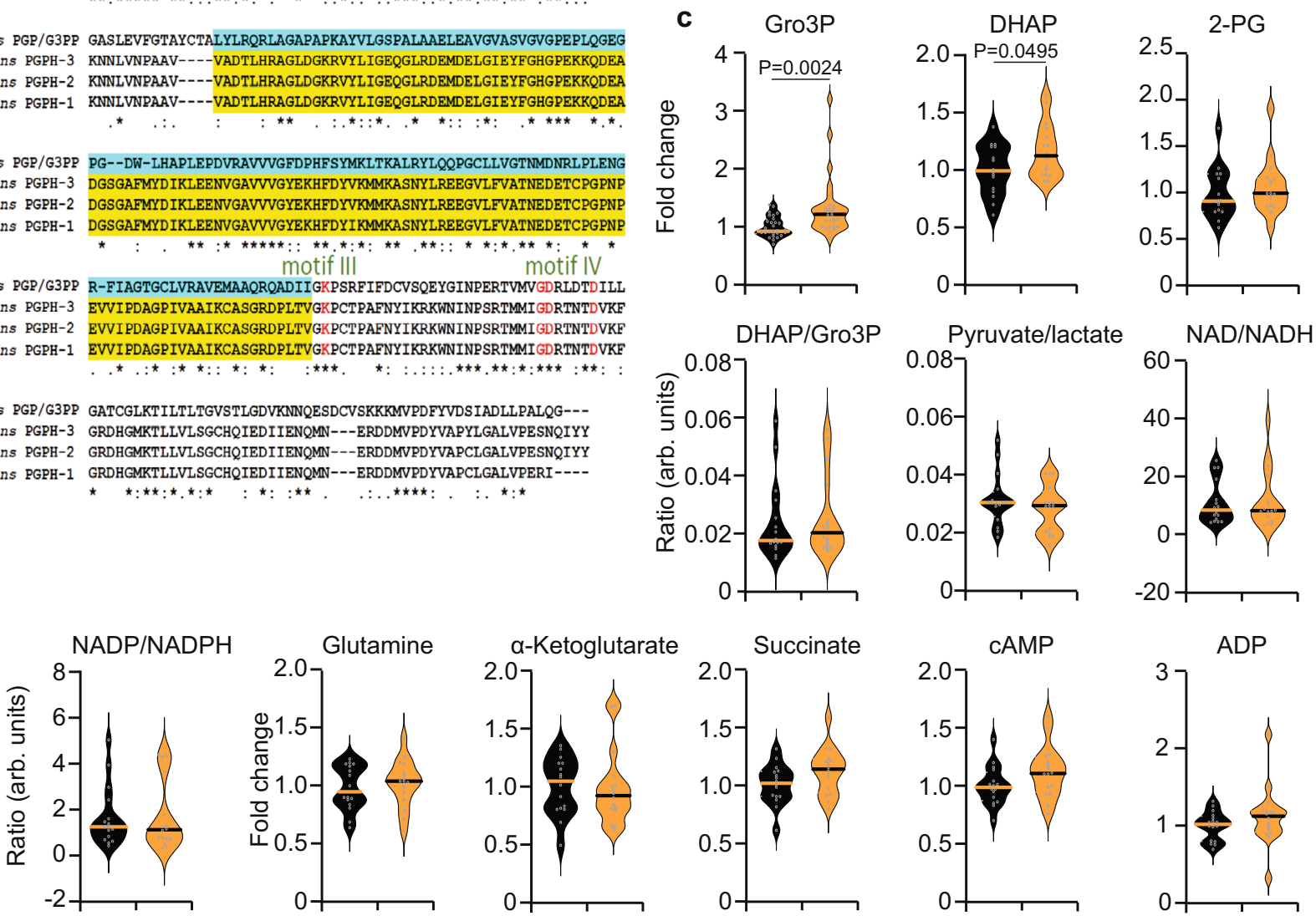

Oil red $\mathrm{O}$

d

e
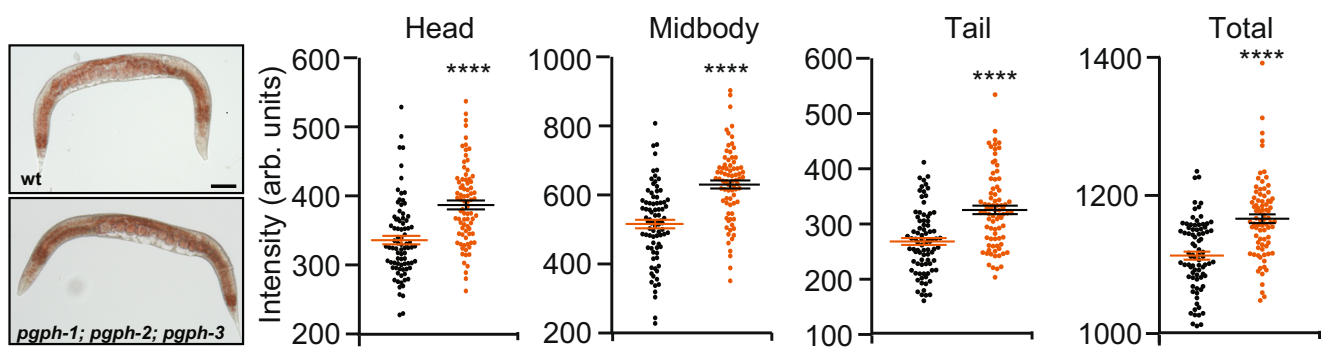

f

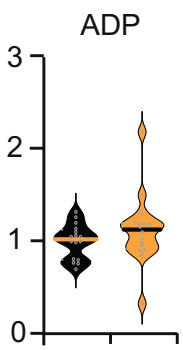

TG

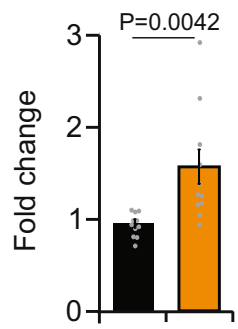

wt

pgph-1; pgph-2; pgph-3

Fig. 1 C. elegans harbors three homologs of mammalian PGP/G3PP with glycerol-3-phosphate phosphatase function. a Sequence alignment of PGP/ G3PP was performed using Clustal Omega. Regulatory motifs are indicated and the cap domain is highlighted. b Glycerol content in wild-type (WT) and pgph-1; pgph-2; pgph-3 synchronized L4/young adult animals grown on NGM plates. Data represent mean \pm SEM, three biological replicates, and three independent experiments. c Violin plots of relative metabolite levels and ratios of some metabolites in WT and pgph-1; pgph-2; pgph-3 synchronized L4/ young adult animals grown on NGM plates. Lines in violin plots denote the median of the analyzed groups and gray circles indicate individual data points (four biological replicates per group per independent experiment and four independent repeats). d, e Oil red O staining and quantification in WT and pgph1; pgph-2; pgph-3 mutant 1-day adult animals. Data represent mean \pm SEM from three independent experiments (WT, $n=83$ and pgph-1; pgph-2; pgph-3, $n=81$ ). Scale bars represent $50 \mu \mathrm{m}$. f Triglyceride levels in WT and pgph-1; pgph-2; pgph-3 1-day adult synchronized animals. Data represent mean \pm SEM, three biological replicates per group per independent repeat, and three independent experiments. In all figures, ${ }^{\star \star \star \star} P<0.0001$ and $P$-values were obtained by student's two-tailed t-test. Data are provided as a Source Data file. 
a

\section{Lipogenesis from Gro3P}
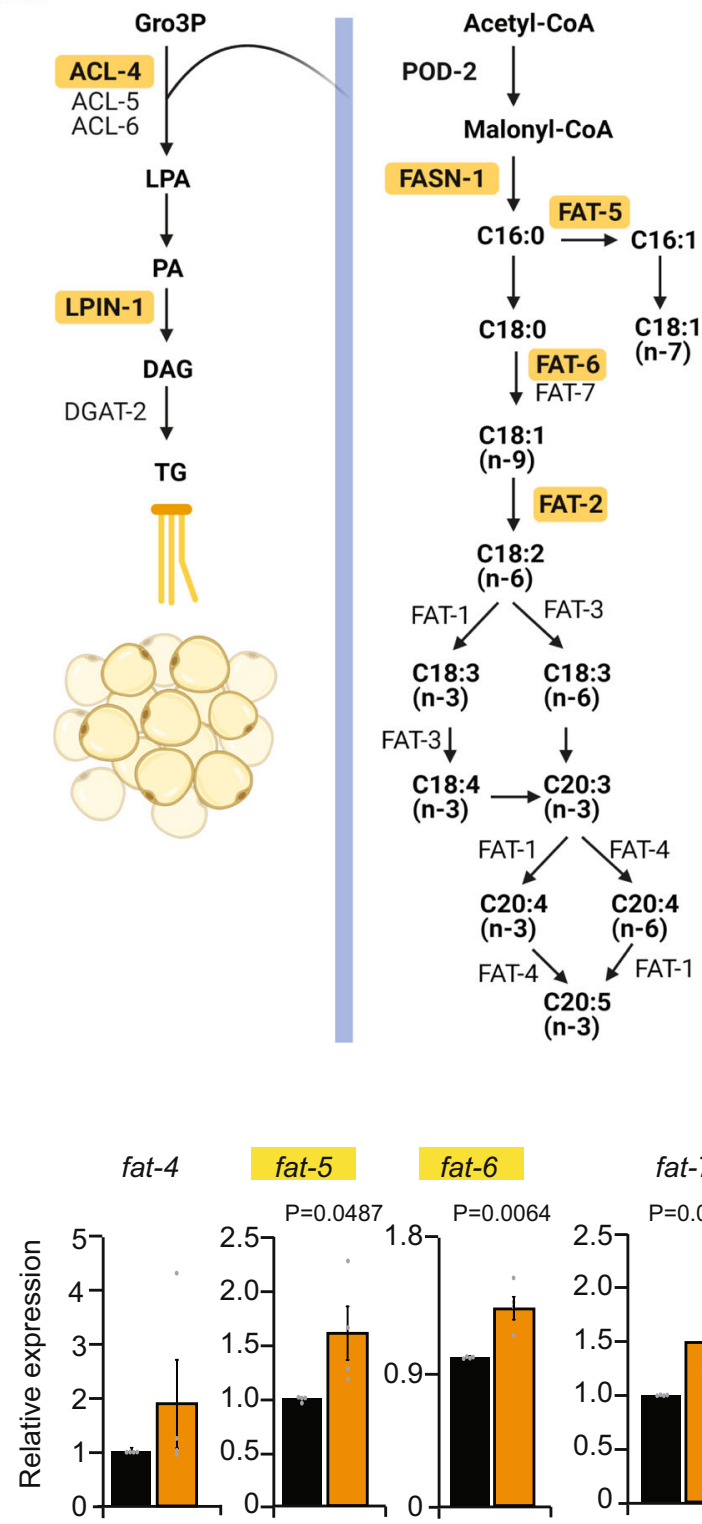

b

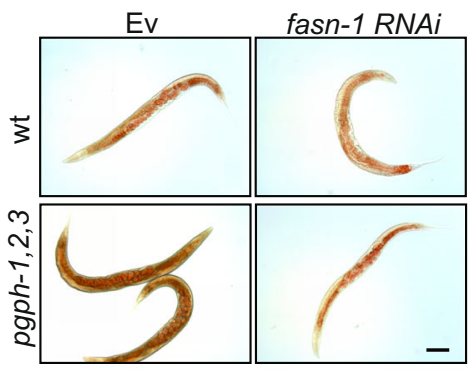

C

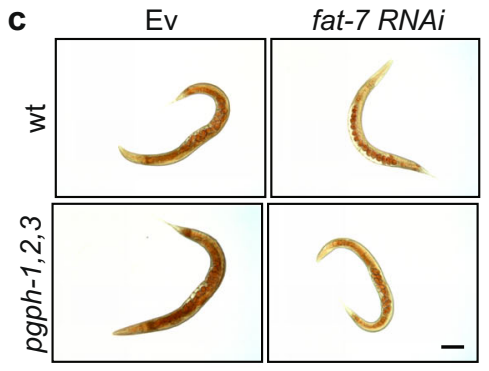

d Lipogenesis genes$$
\text { pod-2 fasn-1 }
$$

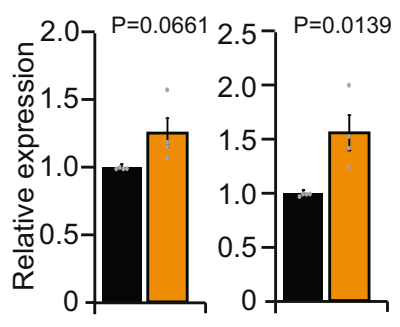

fat-7
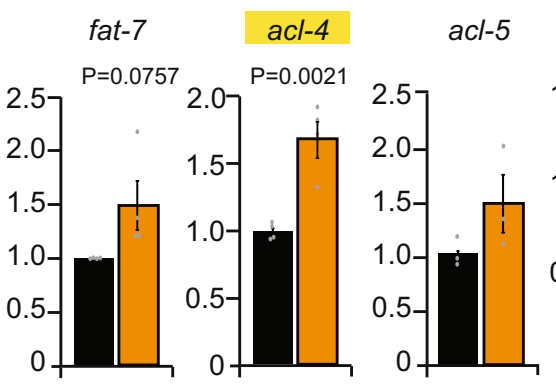

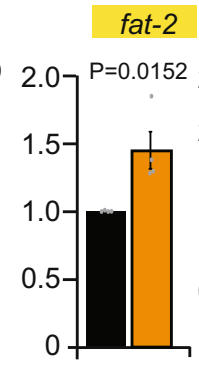

wt pgph-1; pgph-2; pgph-3
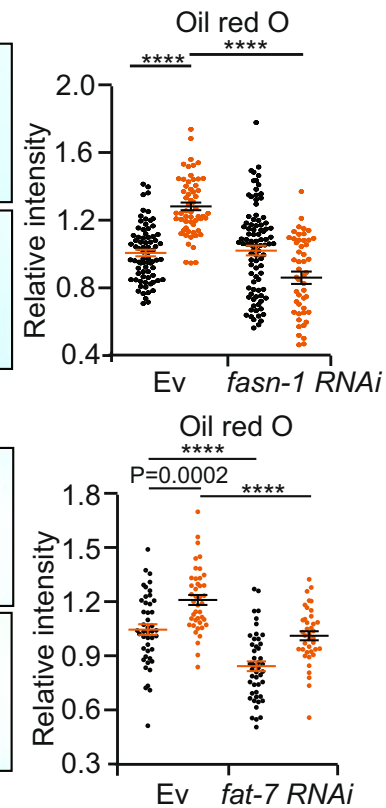

Ev fat-7 RNAi

fat-3

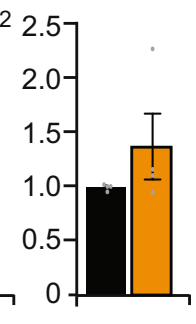

Ipin-1 dgat-2

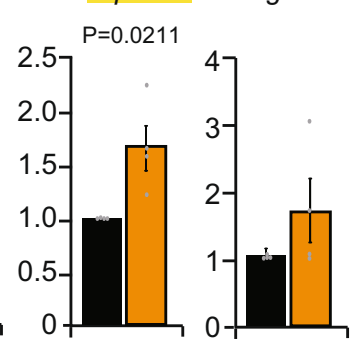

wt

Fig. 2 Enhanced lipogenesis is required for the fat accumulation in triple pgph mutant animals. a Scheme showing the lipogenesis pathway in $C$. elegans highlighting in yellow the genes that are significantly induced in pgph-1; pgph-2; pgph-3 mutant animals at basal level. b, c Oil red O staining and quantification in WT and pgph-1; pgph-2; pgph-3 mutant 1-day adult animals treated or not with fasn-1 RNAi (b) or fat-7 RNAi (c). Scale bars indicate $50 \mu$ m. Sample numbers for b: $n=76$ (WT-Ev), $n=59$ (pgph-1; pgph-2; pgph-3-Ev) and $n=93$ (WT-fasn-1 RNAi), $n=50$ (pgph-1; pgph-2; pgph-3 - fasn-1 RNAi). Sample numbers for $\mathbf{c}: n=45$ (WT-Ev), $n=42$ (pgph-1; pgph-2; pgph-3-Ev), and $n=47$ (WT-fat-7 RNAi), $n=39$ (pgph-1; pgph-2; pgph-3-fat-7 RNAi). Data represent mean \pm SEM from two independent experiments. P-values were obtained by one-way ANOVA with the Bonferroni test. $\mathbf{d}$ Relative expression of indicated genes in WT animals and pgph-1; pgph-2; pgph-3 synchronized young adult animals. Genes that are significantly induced are highlighted in yellow. Data represent mean \pm SEM, $n=4$ independent experiments. $P$-values were obtained by the student's two-tailed $t$-test. Significance in all figures: $\star \star \star \star P<0.0001$. Data are provided as a Source Data file.

(Fig. 2b). Also, fat-7 RNAi significantly reduced the lipid droplets in triple pgph mutant animals (Fig. 2a, c). Importantly, expression of many lipogenesis genes including fasn-1, fat-2, fat-5, fat-6, acl4 , and lpin-1 were significantly increased in triple pgph mutant animals at normal growth conditions supporting that the lipogenesis branch is activated in these mutants (Fig. 2d). We also measured the expression of several lipolysis genes to see whether lipolysis is also altered in the triple pgph mutant animals but did not observe a significant difference in any of the tested genes (Supplementary Fig. 2). Overall, our data support the view that 
suppression of PGPH enzymes increases worm fat levels via lipogenesis.

Salt stress-induced $p g p h$ gene expression is required for glycerol production and resistance to hyperosmotic stress. We investigated whether PGPH loss abrogates glycerol production and salt stress adaptation under hyperosmotic stress, known to enhance glycerol synthesis ${ }^{31,38}$. Hyperosmotic $(400 \mathrm{mM} \mathrm{NaCl})$ stress markedly increased the mRNA expression of $p g p h-1, p g p h-$ 2, and $p g p h-3$ to a similar extent as $g p d h-1$ (Fig. 3a). The gene expression of $p g p h-1$ has been previously shown to increase with high osmolarities and in C. elegans $d p y-7$ mutant animals ${ }^{39}$. Transcriptional fluorescent reporters of $p g p h-2$ and $p g p h-3$ were also activated following $\mathrm{NaCl}$ stress (Supplementary Fig. 3a, b). Importantly, increased glycerol levels under hyperosmotic stress occurred only in wild-type (WT) animals and not in triple pgph mutants suggesting that PGPH enzymes are strictly required for glycerol synthesis under salt stress (Fig. 3b). Accordingly, Gro3P levels decreased in WT but not in the triple pgph mutant nematodes following salt stress, indicating a direct hydrolysis of Gro3P to glycerol in WT worms. Moreover, the increased Gro3P and DHAP levels following $\mathrm{NaCl}$ treatment in triple $p g p h$ mutant animals demonstrate that the rapid conversion of Gro3P to glycerol does not occur if PGPH enzymes are deleted (Fig. 3c). Loss of PGPH did not change 2-PG levels both at basal level and following salt stress (Fig. 3c). Hyperosmotic stress decreased lactate levels and increased the levels of some amino acids including glutamine, alanine, and aspartate. However, our metabolomics analysis revealed that salt stress-related metabolite perturbations are not associated with PGPH loss (Fig. 3c and Supplementary Fig. 3c). Because PGPH loss prevents glycerol production, an organic osmolyte critical for adaptation to hyperosmotic stress, we anticipated that the triple pgph mutant animals would show hypersensitivity to salt stress. Indeed, there was a pronounced decline in the survival of $p g p h$ mutant nematodes in the presence of $\mathrm{NaCl}$ (Fig. 3d and Supplementary Data 1). Overall, these data suggest that PGPH enzymes are required for glycerol synthesis and resistance to hyperosmotic stress in the worm.

PGPH enzymes are transcriptionally regulated by ELT-2 / GATA transcription factor. To determine how PGPH enzymes are transcriptionally regulated upon stresses that require a fast glycerol production such as hyperosmotic stress, we conducted an RNAi screen using the transcription factor RNAi library. Using GFP transcriptional reporter strains for $p g p h-2$ and $p g p h-3$, we individually suppressed the expression of 405 transcription factors by RNAi feeding and screened for reduced GFP fluorescence (Supplementary Fig. 4). We identified 7 transcription factors capable of regulating the promoter activity of both $p g p h-2$ and pgph-3 following salt treatment, fkh-6, elt-2, ets-5, unc-120, nhr89, taf-7.1, and daf-16 (Supplementary Fig. $4 \mathrm{~b}$ and Supplementary Data 7). ELT-2 is required for osmotic stress resistance and the regulation of $g p d h-1$ involved in Gro3P production ${ }^{32}$. Confocal imaging of young adult $p g p h-2$ and $p g p h-3$ transcriptional reporter (GFP expressing) strains exposed to elt-2 RNAi revealed that RNAi-knockdown of elt-2 strongly suppressed both $p g p h-2$ and pgph-3 promoter activities (Supplementary Fig. 4c). These results identify transcriptional regulators of $p g p h-2$ and $p g p h-3$ expression following salt stress and a role of ELT-2 in pgph gene regulation and glycerol production.

Glucose increases $p g p h-1 / p g p h-2$ expression and glycerol release and enhances survival to salt stress. Glucose feeding increases glycerol production in C. elegans ${ }^{14}$. In order to ascertain whether PGPH enzymes are necessary for glycerol synthesis in general, or only under specific conditions such as salt stress, WT animals were exposed to $2 \%$ glucose and glycerol formation was measured. Glucose increased the transcription of pgph-1 and pgph-2 genes (Fig. 4a) and led to a better survival following salt stress (Fig. 4d). Importantly, loss of PGPH enzymes suppressed the glucose-induced glycerol production demonstrating that these enzymes are required for glycerol synthesis from glucose (Fig. 4b). Strikingly, prior exposure of WT animals to glucose followed by treatment with $400 \mathrm{mM} \mathrm{NaCl}$ strongly induced glycerol production, whereas the triple $p g p h$ mutant animals displayed only limited glycerol production under these conditions (Fig. 4b). Gro3P and DHAP levels accumulated in triple pgph mutant animals following glucose stress while no major changes were seen in other metabolites (Fig. 4c and Supplementary Fig. 5a). Accordingly, WT animals pretreated with $2 \%$ glucose displayed better survival under hyperosmotic stress in comparison to the non-treated WT group. Glucose excess that favors glycerol production, protected the double and triple pgph mutants from salt stress (Fig. 4d and Supplementary Data 1), suggesting that when G3PP activity is deleted some glycerol can still be produced upon salt stress via the classical lipogenesis-lipolysis pathway. These results may also imply that glucose may increase the survival to hyperosmotic stress by mechanisms beyond glycerol synthesis such as increased energy, production of other osmolytes such as sorbitol and other possibilities. In fact, the short-term glucose exposure may increase the osmolarity in the plates and precondition the worms to hyperosmotic stress, thereby activating protective mechanisms such as the unfolded protein response and ER hormesis. Overall the results demonstrate that: (a) glycerol formation correlates with PGPH/G3PP expression; (b) PGPH enzymes catalyze Gro3P hydrolysis under control, salt stress, and glucotoxic conditions; and (c) G3PP activity is required for resistance to hyperosmotic stress via glycerol production.

pgph-2 is the primary PGPH isozyme contributing to Gro3P hydrolysis. To determine which of the three PGPH isoenzymes plays a prominent role in the worm glycerol production, we used a comparative approach between a double pgph mutant (pgph-2; $p g p h-3)$ strain generated by CRISPR-CAS9, the triple $p g p h$ mutant strain (pgph-1; pgph-2; pgph-3) and single pgph-2 and $p g p h-3$ mutant worms. The specific role of $p g p h-1$ was not directly investigated with a single mutant strain because the high sequence identity between pgph-1 and the other two isoforms prohibited the generation of a specific CRISPR deletion mutant. However, the genetic comparison of the survival to hyperosmotic stress, glycerol levels, and Gro3P measurements in double and triple pgph mutant animals implied a less important role for pgph-1 for the following reasons. First, we did not observe any defect in physiological parameters including pharyngeal pumping, egg laying, as well as brood size in the double pgph mutant strain (Supplementary Fig. 6a-c), similar to what we have noticed with the triple pgph mutant strain (Supplementary Fig. 1a-c). Under normal growth conditions, glycerol levels were only significantly reduced in the triple $p g p h$ mutant animals with a reduction trend in double pgph mutant (Supplementary Fig. 6d). As previously demonstrated (Fig. $3 \mathrm{~b}$ and Fig. 4b), WT animals exhibited a dramatic increase in glycerol levels following salt stress, glucose stress, or the two stresses simultaneously (Supplementary Fig. 6e, f). However, the extent of reduction in glycerol levels was similar between double and triple $p g p h$ mutant animals under these conditions, indicating that lack of $p g p h-1$ does not cause a stronger defect in glycerol synthesis (Supplementary Fig. 6e, f). Accordingly, Gro3P levels in double and triple 

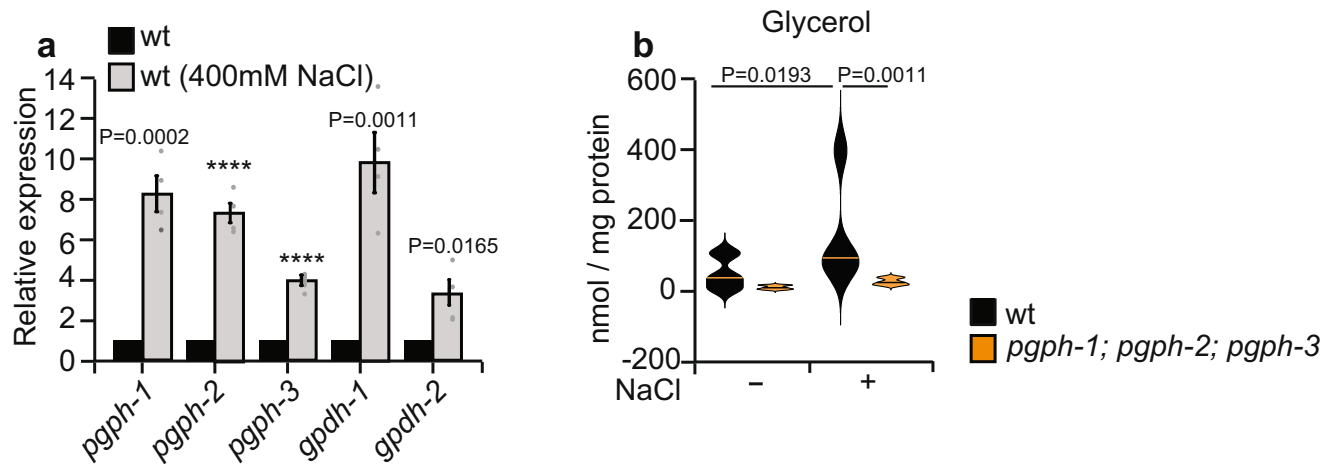

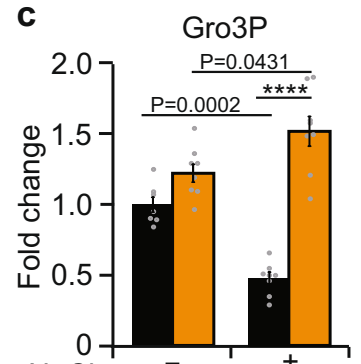

$\mathrm{NaCl}$
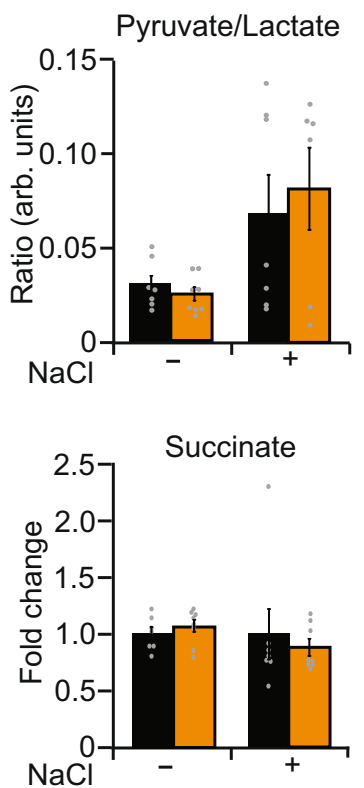

\section{DHAP}

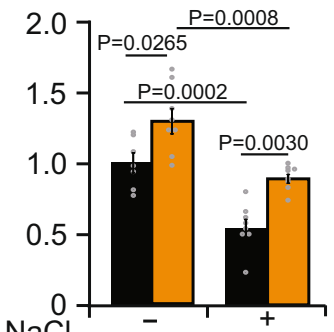

$\mathrm{NaCl}$
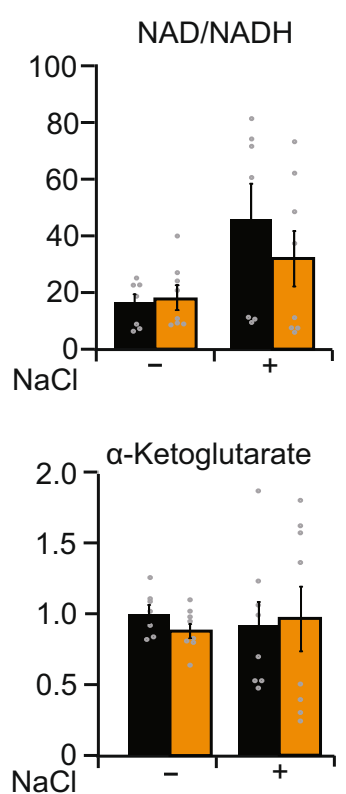
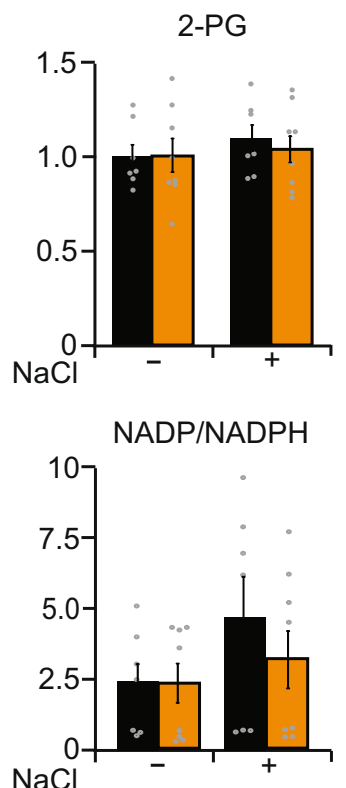

$\mathrm{NaCl}$

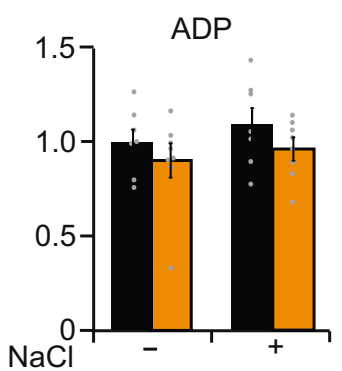

DHAP/Gro3P
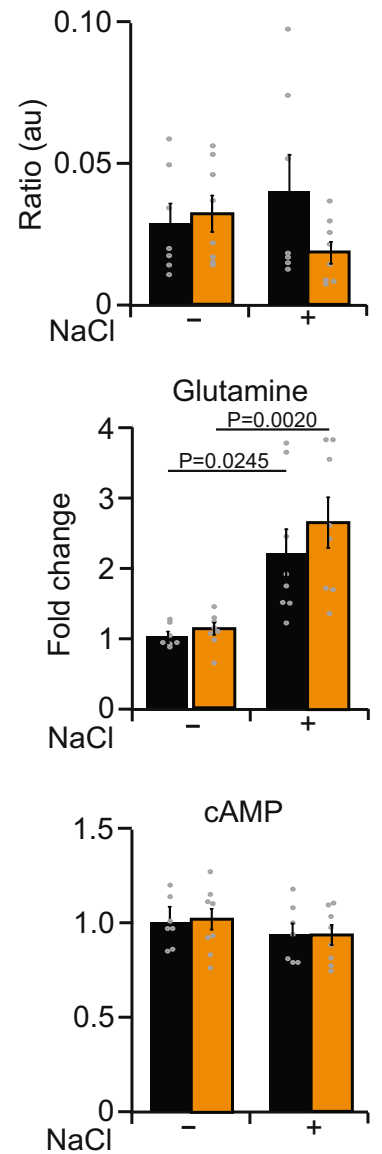

d

Hyperosmotic stress

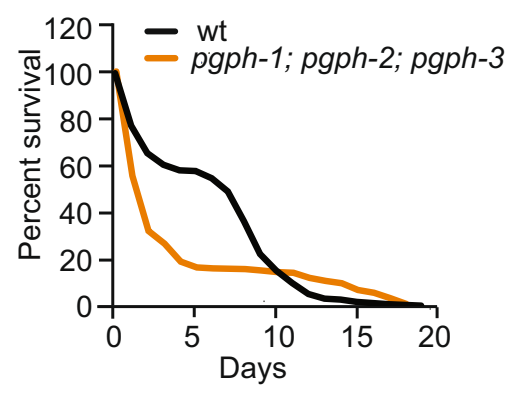

pgph mutant animals were not significantly different under normal growth conditions or following hyperosmotic stress or excess glucose (Supplementary Fig. 6g-i). Oil red O staining revealed a similar increase in fat levels in the double and triple $p g p h$ mutants, driven by elevated lipogenesis (Supplementary Fig. 6j). Moreover, double and triple $p g p h$ mutant animals were equally

hypersensitive to hyperosmotic stress (Supplementary Fig. 6k-m). Pre-treatment of the worms with $2 \%$ glucose or $1 \%$ glycerol increased the survival of the animals to $400 \mathrm{mM} \mathrm{NaCl}$ (Fig. $4 \mathrm{~d}-\mathrm{f}$ and Supplementary Fig. $6 \mathrm{n}-\mathrm{p}$ and Supplementary Data 1) and both double and triple pgph mutant animals pretreated with $2 \%$ glucose or $1 \%$ glycerol were hypersensitive in comparison to the 
Fig. 3 PGPH/G3PP is required for glycerol production to allow resistance to hyperosmotic stress. a Relative expression of indicated genes in WT animals treated or not with $400 \mathrm{mM} \mathrm{NaCl}$ for $3 \mathrm{~h}$. Data represent mean $\pm \mathrm{SEM}$, four independent experiments. $P$-values are obtained by unpaired two-tailed student's $t$-test. b Violin plots representing glycerol levels of WT and pgph-1; pgph-2; pgph-3 synchronized L4/young adults exposed or not to 400 mM $\mathrm{NaCl}$ for $3 \mathrm{~h}$. In all groups, $n=9$. Plots are generated with three biological replicates per group per independent repeat and a total of three independent experiments. Lines in the violin plots represent median of corresponding groups. P-values are obtained by one-way ANOVA with the Bonferroni test. $\mathbf{c}$ Relative metabolite levels and ratios of some metabolites in WT and pgph-1; pgph-2; pgph-3 synchronized L4/young adult animals grown on NGM plates and exposed or not to $400 \mathrm{mM} \mathrm{NaCl}$ for $3 \mathrm{~h}$. Data represent mean $\pm \mathrm{SEM}, n=8$, four biological replicates per group per independent repeat and two independent experiments. $P$-values are obtained by one-way ANOVA with the Bonferroni test. $\mathbf{d}$ Survival curves of indicated strains to $400 \mathrm{mM} \mathrm{NaCl}$. $P$ values are obtained by two-sided Mantel-Cox comparisons. Number of separate experiments, animals, and detailed statistics are shown in Supplementary Data 1. Significance in all figures: ${ }^{\star \star \star \star} P<0.0001$. Data are provided as a Source Data file.

treated WT group (Fig. 4d-f and Supplementary Fig. 6n-p and Supplementary Data 1). However, triple pgph mutant animals lacking pgph-1 in comparison to the double mutant animals were slightly more hypersensitive to salt stress, suggesting that $p g p h-1$ may play a small role under conditions with excess Gro3P precursors (Fig. $4 \mathrm{f}$ and Supplementary Fig. 6p). Overall, the data indicate that $p g p h-1$ likely plays a minor role in glycerol synthesis, fat accumulation, and survival to hyperosmotic stress.

Using single mutant strains, loss of $p g p h-2$ alone in two different alleles mimicked the salt stress hypersensitivity observed in the double and triple pgph mutant animals (Supplementary Fig. 6k and Supplementary Data 1). In contrast, pgph-3(tm3391) mutant animals were not sensitive to salt stress (Supplementary Fig. 61 and Supplementary Data 1). Moreover, in 1-day adult animals, $p g p h-2$ was found to be transcriptionally activated in the intestine following $\mathrm{NaCl}$ stress, while $p g p h-3$ activation occurred mostly in embryos (Supplementary Fig. 3b). Overall, our data point towards a more prominent role for $p g p h-2$ and a less important role for $p g p h-1$ and $p g p h$-3. Our results demonstrate that PGPH isoenzymes in the worm, in particular $p g p h-2$, act as Gro3P phosphatase for glycerol synthesis under both normal conditions and under salt stress and are required for survival to hyperosmotic stress.

PGPH loss shortens lifespan and healthspan in normal and high glucose conditions. Excess glucose is known to induce glucotoxicity as well as early onset of aging-related abnormalities $^{14}$. Loss of PGPH enzymes, via either RNAi or double and triple deletion mutations, significantly reduced the median lifespan of C. elegans (about 12\%, see Supplementary Data 2), supporting the view that it plays a protective role in aging and healthy aging (Fig. 5a, b and Supplementary Fig. $7 \mathrm{~d}$ and Supplementary Data 2). We next investigated the influence of PGPH loss on movement behaviors, an index of healthy aging, in WT and pgph mutants. Locomotion depends on the integrity of the worm neuromuscular function ${ }^{40-42}$, and a decline in movement reflects a deterioration in health, normally observed after day 5 in WT animals ${ }^{43-47}$. Motility of the aging pgph mutant animals in terms of number of body bends per second, speed, and the peristaltic speed, deteriorated faster as compared to WT nematodes (Fig. 5c-e and Supplementary Fig. 7e, f). Recent reports indicate that increased stress resistance and improved locomotion in aging C. elegans animals are indicative of extended health span and healthy aging ${ }^{46}$. We measured the ability of the double and triple pgph mutant animals to resist various stresses. Reduced PGPH enzymes expression via RNAi increased the susceptibility to oxidative stress, and delayed recovery after $24 \mathrm{~h}$ hypoxia and $16 \mathrm{~h}$ cold stress (Supplementary Fig. $7 \mathrm{a}-\mathrm{c}$ and Supplementary Data 4-6). Obesity linked to excess carbohydrate reduces lifespan and healthspan in many organisms ${ }^{14,48-52}$. We next determined whether loss of PGPH exacerbates the lifespan decline caused by excess glucose. Loss of all three PGPH enzymes significantly reduced median lifespan (about 7-16\%; see
Supplementary Data 3) upon glucose treatment, supporting a protective role for PGPH enzymes in the presence of excess glucose (Fig. 5f, g and Supplementary Fig. 7g). Accordingly, movements deteriorated faster during aging in pgph mutant animals in comparison to WT upon glucose feeding (Fig. 5h-j and Supplementary Fig. 7h, i). These results support a role for PGPH enzymes in aging, particularly healthy aging, as well as in combating various stresses.

pgph-2 overexpression improves healthspan and decreases fat content. There is a growing interest in interventions that compress the years of decay and disease and extend the years of healthy living, rather than extending maximal lifespan ${ }^{25,52-54}$. Considering that pgph mutant worms showed accelerated aging and declined healthspan in normal as well as excess glucose conditions, along with susceptibility to various stresses, we investigated whether the overexpression of PGPH enzymes protects against the above parameters. We generated transgenic worms overexpressing either pgph-2 or pgph-3 and assessed lifespan and healthspan parameters. Overexpression of $p g p h-2$ led to a small but significant increase in median lifespan in three separate transgenic lines (about 6\%; Supplementary Data 2) under control non-stress condition (Fig. 6a and Supplementary Fig. 8a, b). The three transgenic lines showed mild overexpression of no more than three fold (Supplementary Fig. 7j). Video imaging of transgenic animals overexpressing pgph-2 revealed that they maintain a younger age-associated locomotion behavior for a longer time in comparison to Control animals WT animals (Supplementary movies 1-4 taken at day 9). The number of body bends per second, speed, and peristaltic speed were significantly higher in the $p g p h-2$ overexpressing animals with increasing age, supporting a role for this enzyme in the prevention of age-related decline in motor function (Fig. 6b-d and Supplementary Fig. 8c-f).

Dietary restriction in C. elegans is known to reduce body size and decrease fat content ${ }^{55}$. Because PGPH hydrolyzes Gro3P, which is the backbone for the synthesis of glycerolipids, we anticipated that pgph-2 overexpressing worms would display decreased lipogenesis and accumulate less fat with age. Indeed, pgph-2 overexpression reduced body length and mean body surface area (Fig. 6e, f and Supplementary Fig. 8g, h) and decreased fat content in three separate transgenic lines (Fig. 6h, i and Supplementary Fig. 8k). C. elegans feeding depends on the rhythmic contraction of the pharynx known as pharyngeal pumping, a primary indicator of food intake. Pharyngeal pumping rates in $p g p h-2$ overexpressing animals were unchanged demonstrating that the decreased fat content is not due to a decrease in calorie intake (Fig. $6 \mathrm{~g}$ and Supplementary Fig. 8i, j). Moreover, egg laying and brood size were not altered in $p g p h-2$ overexpressing lines suggesting that the extension in healthspan is not at the expense of energy spent on reproduction (Fig. 6j, $\mathrm{k}$ and Supplementary Fig. 81, m). Similar experiments carried out with animals overexpressing $p g p h-3$ did not show differences in any of the above-measured phenotypes. Overall, these results indicate that pgph-2 overexpression, but not pgph-3, slightly extends medium 


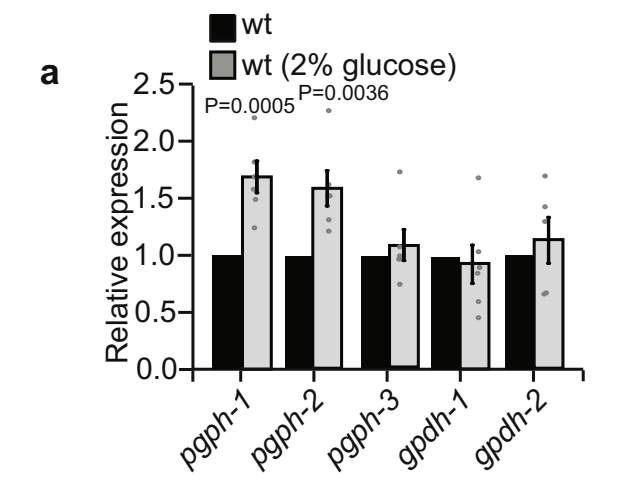

C

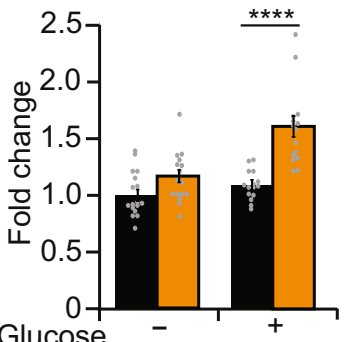

Pyruvate/Lactate

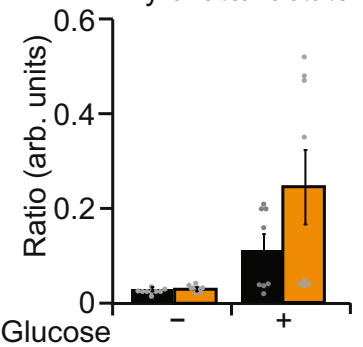

Glucose

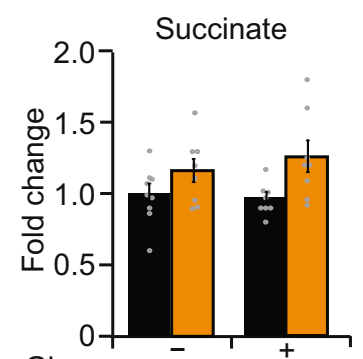

Glucose

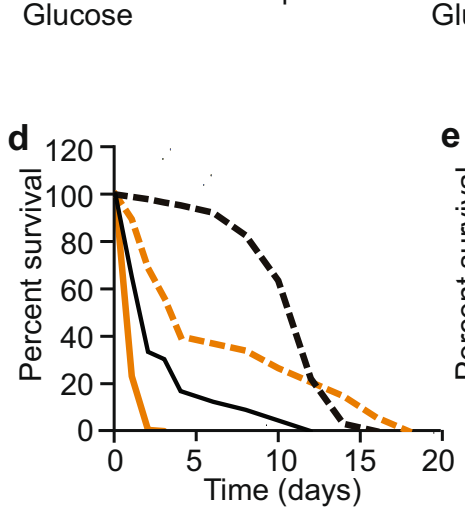

Glucose

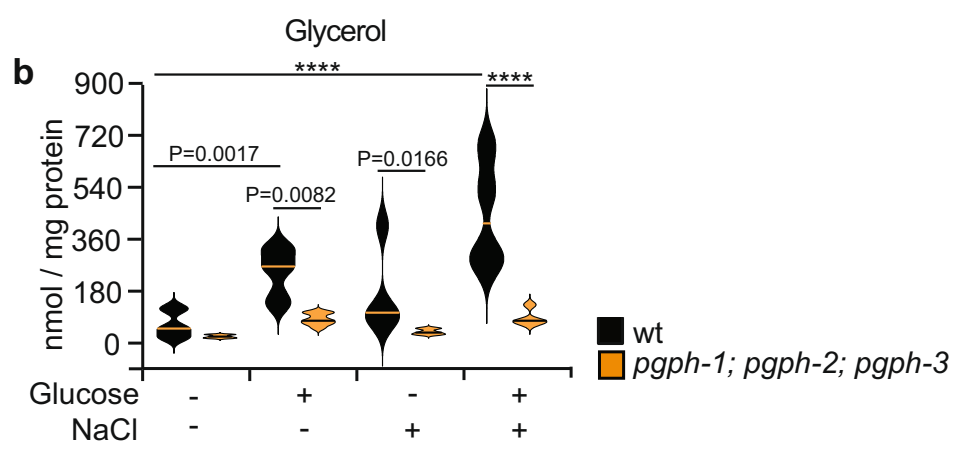

2-PG

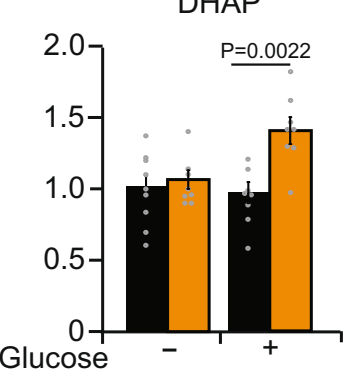

\section{NAD/NADH}
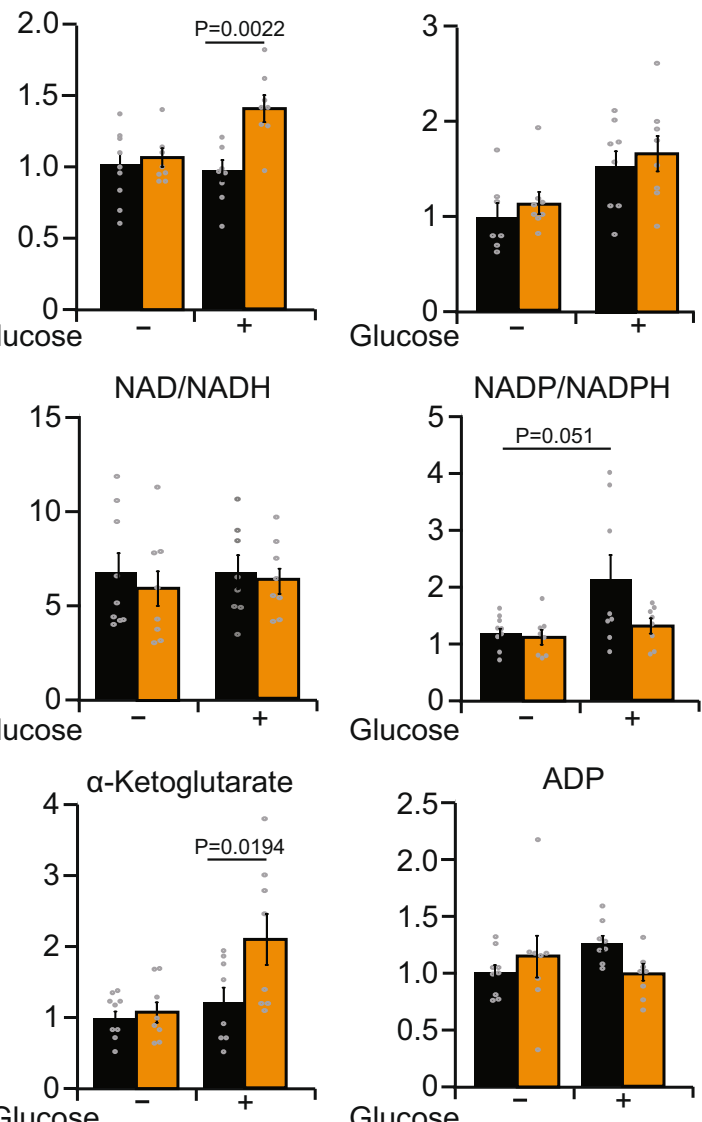

Glucose
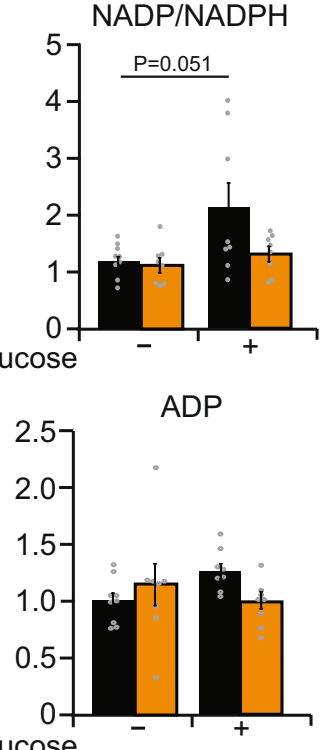

DHAP/Gro3P
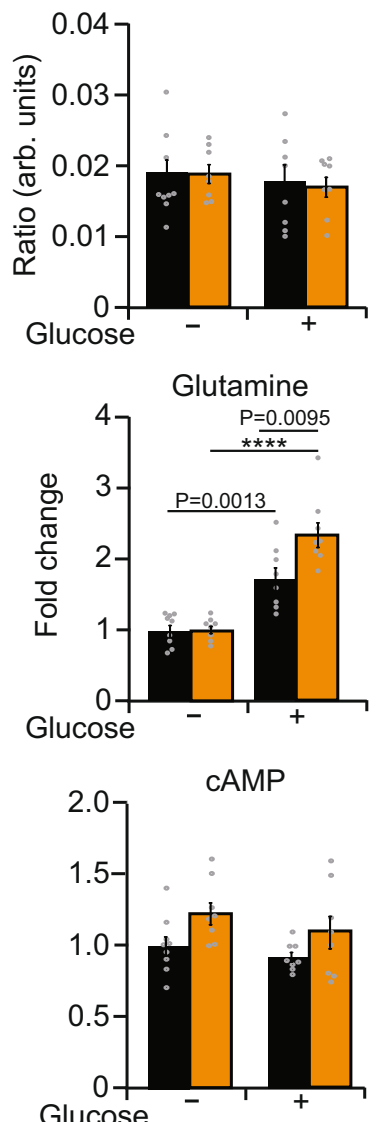

Hyperosmotic stress +/- glucose
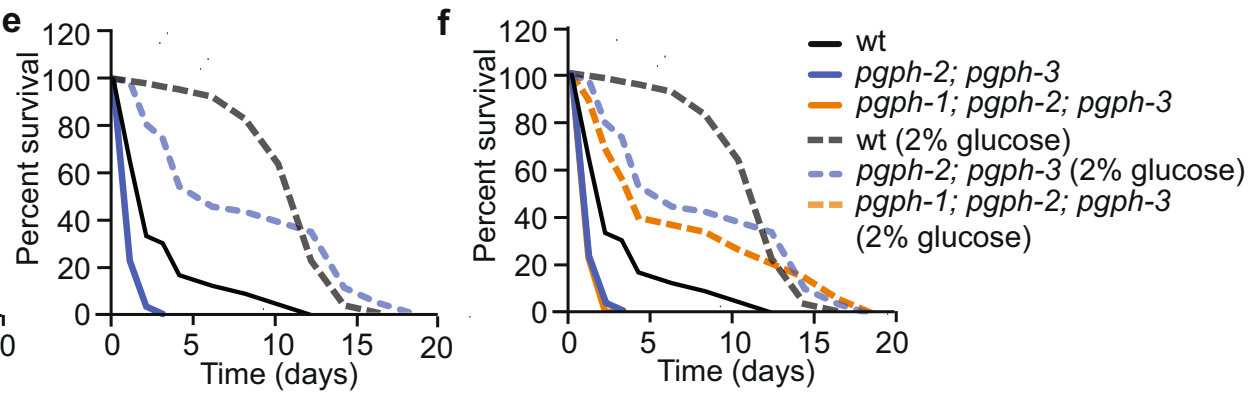

lifespan, but has significant beneficiary effects in delaying age-related decline in locomotor function and in preventing fat accumulation.

pgph-2 overexpression increases lifespan in the presence of glucose and reduces glucose-induced fat deposition via reduced lipogenesis. Three separate transgenic lines overexpressing pgph2 showed a significant increase (about 20\%) in medium lifespan in the presence of glucose (Fig. 7a and Supplementary Fig. 9a, b, Supplementary Data 3). Video imaging of glucose-fed pgph-2 overexpressing animals revealed that they maintained a better locomotion behavior with age (Supplementary movies 5-8 at day 
Fig. 4 Glucose induces pgph-1/pgph-2 gene expression, stimulates Gro3P and glycerol release in a PGPH-dependent manner, and enhances survival to salt stress. a Relative expression of indicated genes in synchronized L4/young adult WT animals grown on NGM plates or plates supplemented with $2 \%$ glucose. Data represent mean \pm SEM, six independent experiments. $P$-values are obtained by two-tailed student's $t$-test. b Violin plots representing glycerol levels of WT and pgph-1; pgph-2; pgph-3 synchronized L4/young adults grown on NGM plates or plates supplemented with $2 \%$ glucose. On the day of the experiment, the two groups were either treated or not (for control) with $400 \mathrm{mM} \mathrm{NaCl}$ for $3 \mathrm{~h}$. Pellets were then collected, washed with $\mathrm{M} 9$ buffer with adjusted osmolarities, and flash frozen in liquid nitrogen for glycerol extraction and quantification. In all groups, $n=9$. Plots are generated with three biological replicates per group per independent repeat and a total of three independent experiments. Lines in the violin plots represent the median of corresponding groups. P-values are obtained by one-way ANOVA with the Bonferroni test. c Relative metabolite levels and ratios of WT and pgph-1; pgph-2; pgph-3 synchronized L4/young adults grown on NGM plates or plates supplemented with $2 \%$ glucose. Data represent mean \pm SEM, $n=8$ biological replicates, four biological replicates per group per independent repeat, and two independent experiments. $P$-values are obtained by one-way ANOVA with the Bonferroni test. d-f Survival curves of indicated strains to $400 \mathrm{mM} \mathrm{NaCl}$ pretreated or not with $2 \%$ glucose. Number of separate experiments, animals, and detailed statistics are shown in Supplementary Data 1. P-values were obtained with two-sided Mantel-Cox test. In all figures: ${ }^{\star \star \star \star} P<0.0001$. Data are provided as a Source Data file.

9). Specifically, the number of body bends per second, speed, and peristaltic speed were much improved in the glucose-fed pgph-2 overexpressing animals (Fig. $7 \mathrm{~b}-\mathrm{d}$ and Supplementary Fig. $9 \mathrm{c}-\mathrm{f}$ ). Furthermore, $p g p h-2$ overexpression reduced body length and mean body surface area in glucose excess conditions (Fig. 7e, $\mathrm{f}$ and Supplementary Fig. 9g, h). Glucose supplementation increased fat content in control animals, whereas animals with pgph-2 overexpression displayed lower fat levels that were comparable to control animals grown on normal growth medium (Fig. 7h and Supplementary Fig. 9k). Importantly, the expression of several lipogenesis genes was significantly decreased in pgph-2 overexpressing animals in comparison to the controls, supporting that lipogenesis is negatively regulated in $p g p h-2$ overexpressing animals, specifically under excess glucose conditions (Supplementary Fig. 11a). Also, we did not observe a significant increase in any of the tested lipolysis genes, suggesting that the decrease in fat deposition in $p g p h-2$ overexpressing animals is unlikely driven by an increased lipolysis, especially following excess glucose (Supplementary Fig. 11b).

Animals overexpressing $p g p h-2$ maintained higher pharyngeal pumping rates than control animals, with increasing age, indicating that the decrease in fat deposition is not due to reduced food intake, and that the overexpression of $p g p h-2$ delays age-related decline in food intake (pharyngeal pumping) under glucotoxic conditions (Fig. $7 \mathrm{~g}$ and Supplementary Fig. 9j). Additionally, brood size and egg laying were not altered in pgph-2 overexpressing lines grown in excess glucose conditions indicating that the increased lifespan and healthspan in the glucose-rich medium are not due to a decrease in reproduction (Fig. 7i, j and Supplementary Fig. 9l, m). In contrast, overexpression of pgph-3 alone did alter lifespan and did not improve healthspan parameters in glucose excess conditions (Supplementary Fig. 10).

Interestingly, these beneficial effects of pgph-2 overexpression tend to be more prominent under glucotoxic stress than normal growth conditions (Supplementary Data 9). In fact, a comparison of survival, locomotion parameters, as well as pharyngeal pumping in three transgenic lines overexpressing pgph-2 reveal larger beneficial effects in the presence of glucose in comparison to the absence of glucose (Supplementary Fig. 12). What may be viewed as a potential caveat in these series of experiments on healthy aging parameters in the absence and presence of glucotoxicity should be mentioned. Thus, in $p g p h$-2-overexpression studies, while many of the phenotypes on days 3 and 7 are more prominent in the presence of $2 \%$ glucose, some effects occur for animals without the addition of glucose for many phenotypes at days 3 and 9 . How can this be explained? One possibility is that a relative small increase in $p g p h-2$ overexpression ( 2.5 fold at mRNA level) is enough to have beneficial effects until day 7 in the presence $2 \%$ glucose but to a lesser extent at day 9 when glucotoxicity is more chronic and therefore more deleterious. Indeed, control animals exposed to $2 \%$ glucose have a medium and maximum lifespan of 13 and 20 days, respectively, compared to 16 and 27 days without glucose. Perhaps higher pgph-2 overexpression could lead to higher beneficial effects also at day 9 but this remains to be tested. Overall, enhanced PGPH-2 activity, unlike PGPH-3, protects from age-related decline and fat accumulation in excess glucose conditions.

\section{Discussion}

Here, we used C. elegans as a model to identify the biological functions of G3PP. We demonstrate that C. elegans harbors three PGPH isozymes with G3PP activity in vivo. We also show that PGPH enzymes regulate glycerol and fat metabolism, organismal adaptation to various stresses, in particular hyperosmotic stress and glucotoxicity, as well as healthy aging (Graphical abstract and Fig. 8).

Multiple lines of evidence demonstrate that PGPH acts as a G3PP in vivo in C. elegans. Thus, metabolomic measurements show that both Gro3P and DHAP are increased under normal conditions upon PGPH enzymes deletion and more prominently following salt and glucose stresses, while other metabolites, including 2-PG, are not perturbed. Moreover, pgph double and triple mutant animals show reduced levels of glycerol under basal conditions and are incapable of inducing glycerol production following salt, glucose, or both stresses together. Importantly, we also show that fat levels are increased in triple pgph mutant animals under basal conditions, in accordance with a rise in Gro3P levels.

The role of glycerol in organismal adaptation to hyperosmotic stress is well established. In C. elegans, GPDH which converts DHAP to Gro3P, is induced in response to acute salt stress and is required for glycerol production for hyperosmotic stress

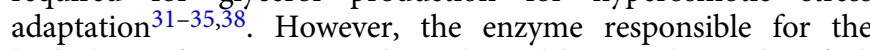
hydrolysis of Gro3P to produce glycerol has not been identified. High osmolarities have been recently shown to induce $p g p h-1$ gene expression ${ }^{39}$. Here, we demonstrate that pgph genes are strongly induced following hyperosmotic and glucotoxic stresses and are required for glycerol production and survival under these stresses.

Besides Gro3P and 2-PG, PGP/G3PP has also been shown to detoxify certain toxic glycolytic side products in cancer cell lines, particularly 4-phosphoerythronate and 2-phospholactate, produced by glyceraldehyde 3-phosphate dehydrogenase and pyruvate kinase, respectively ${ }^{8,56}$. Upon their accumulation following $p g p$ deletion, glycolysis, the pentose phosphate pathway, and ATP production are altered ${ }^{8,57}$. However, our work reveals no change in the levels of key glycolytic and Krebs cycle intermediates, adenine and pyrimidine nucleotides, linked to energy metabolism, except Gro3P and DHAP, with PGPH loss at basal level and 
a

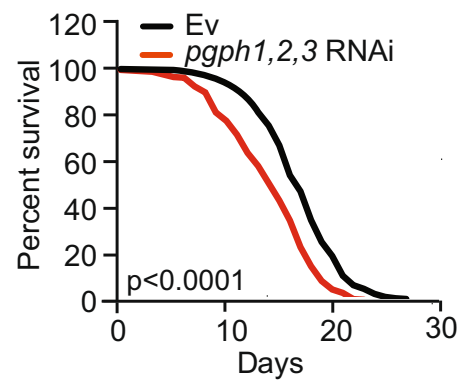

wt $\square$ pgph-1; pgph-2; pgph-3 b

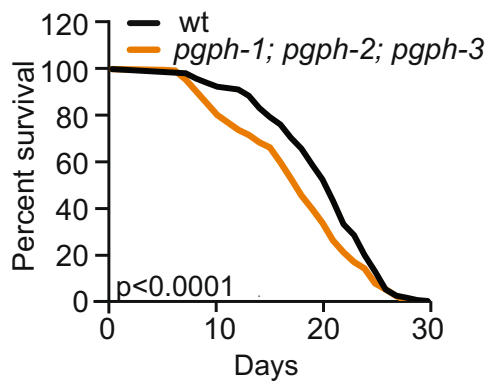

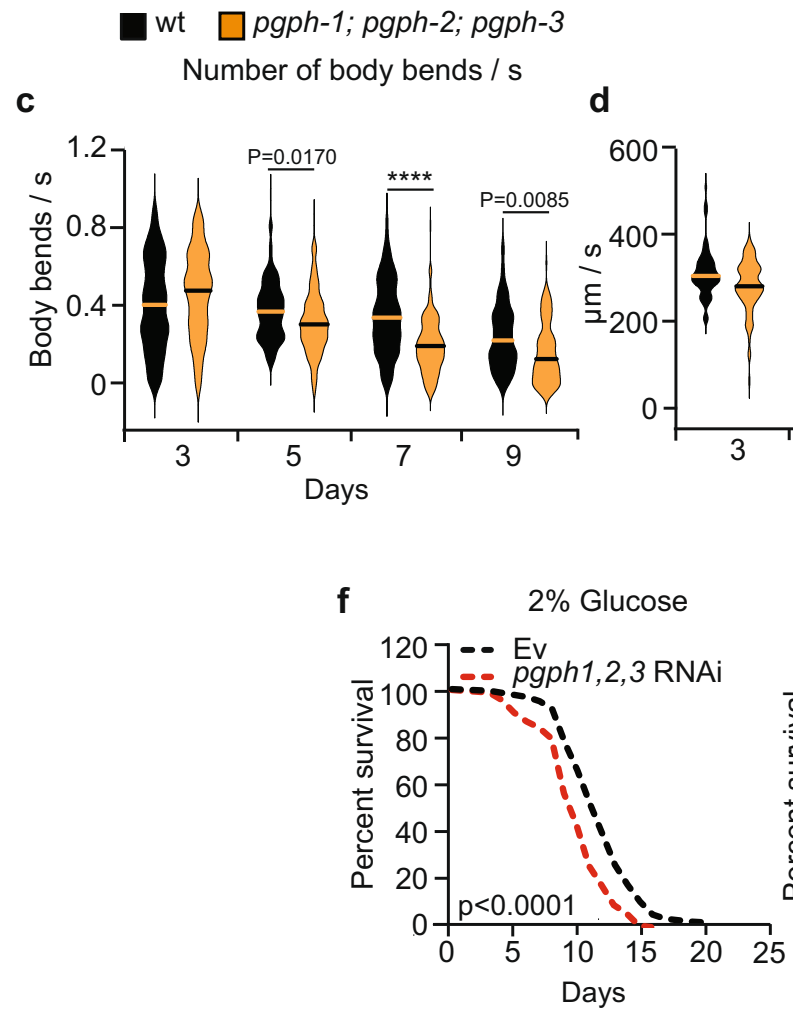

Speed

e

Peristaltic speed

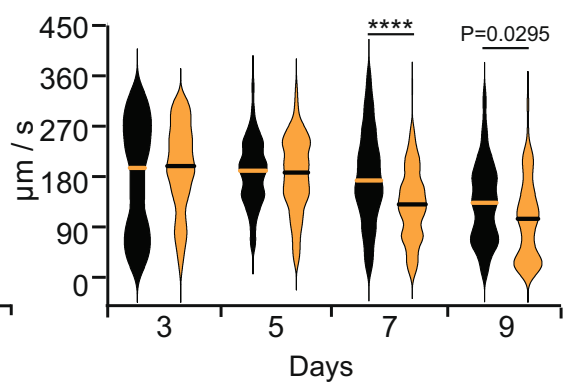

Days

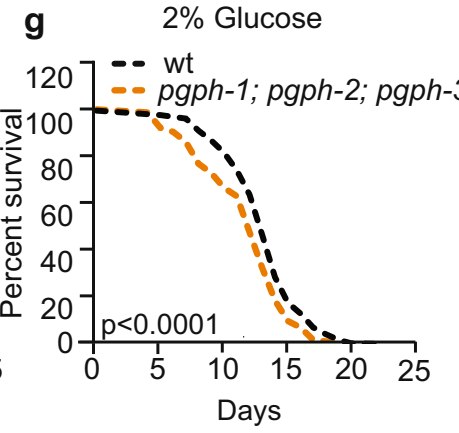

wt $\square p g p h-1 ; p g p h-2 ; p g p h-3$
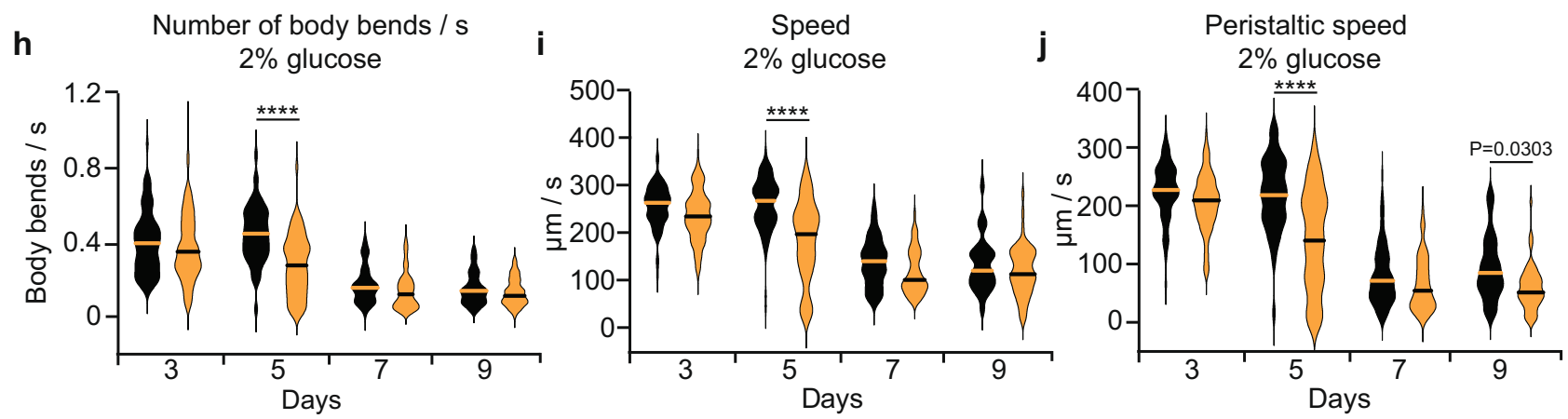

Fig. 5 PGPH loss shortens lifespan and healthspan in normal and high glucose conditions. a Lifespan survival curves of WT animals treated with Ev or pgph RNAi. b Lifespan survival curves of indicated worm strains on NGM plates. c-e Locomotion analysis of worm body bends/s (c), speed (d), and peristaltic speed (e) of indicated worm strains at days 3, 5, 7, and nine of age. Data is represented using violin box plots from three independent experiments with indicated median lines. More than 150 tracks are analyzed per condition and an exact number of tracks is shown in the data source. $\mathbf{f}, \mathbf{g}$ Survival curves of indicated worm strains grown on plates supplemented with $2 \%$ glucose. $\mathbf{h}-\mathbf{j}$ Locomotion analysis of worm body bends/s (h), speed (i), and peristaltic speed (j) of indicated worm strains grown on plates supplemented with $2 \%$ glucose at days 3, 5, 7, and 9 of age. Data is represented using violin box plots from three independent experiments with indicated median lines. More than 150 tracks were analyzed per condition and an exact number of tracks is shown in the data source. Number of separate lifespan and glucotoxicity experiments, animals, and detailed statistics are shown in Supplementary Data 2 and 3. Significance in all figures: ${ }^{\star \star \star \star} P<0.0001$. $P$-values of lifespan and glucotoxicity experiments were obtained by two-sided Mantel-Cox. P-values of locomotion analysis were obtained by one-way ANOVA with the Bonferroni test. Data are provided as a Source Data file. 
a
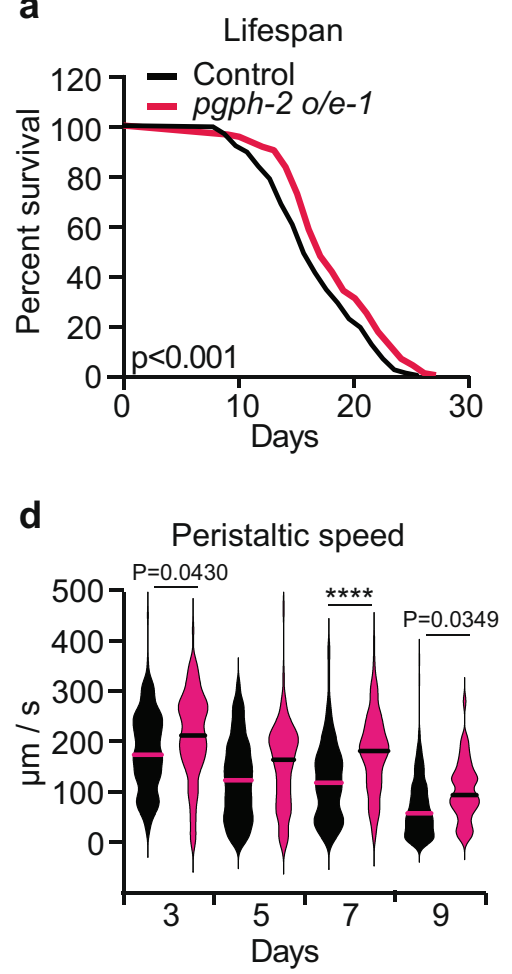

g Pharyngeal pumping

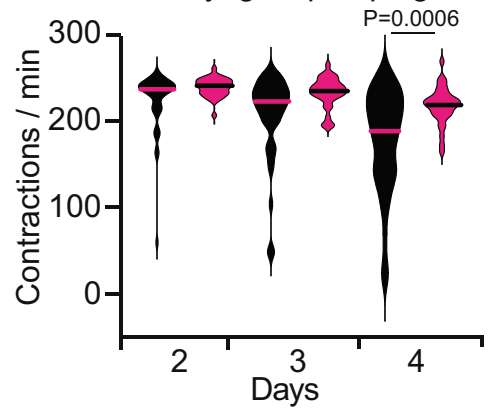

Control

pgph-2 o/e-1

Number of body bends / $\mathrm{s}$
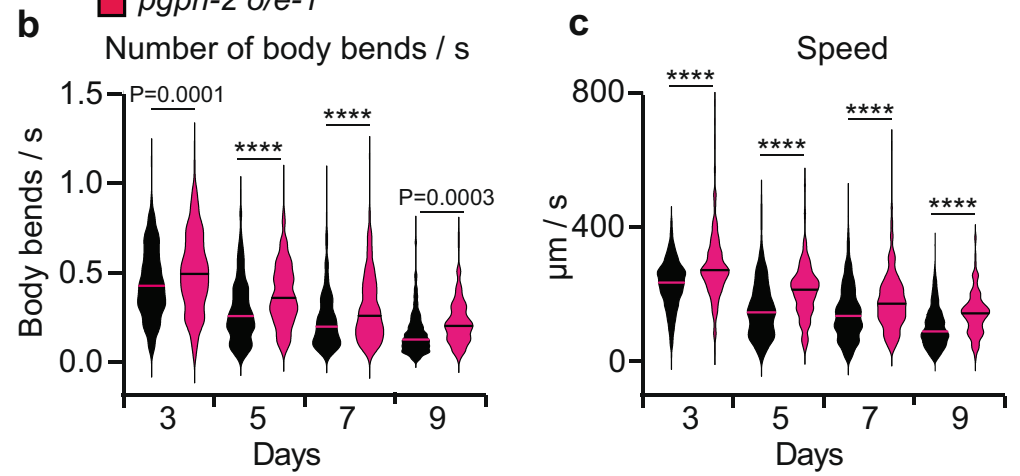

e

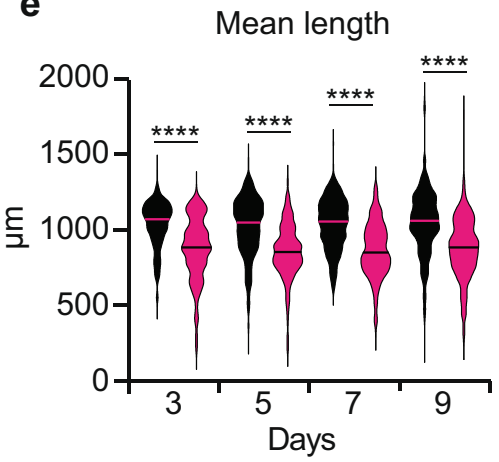

f

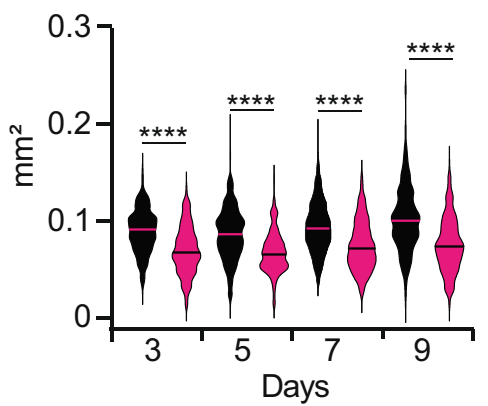

h

Oil red $\mathrm{O}$
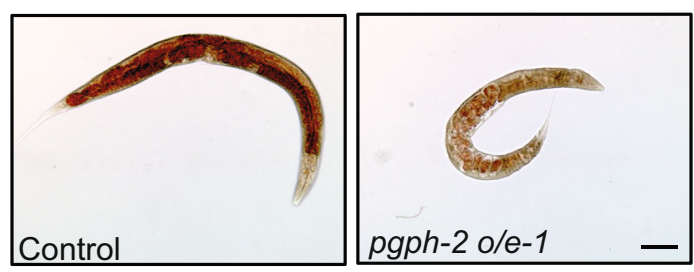

i

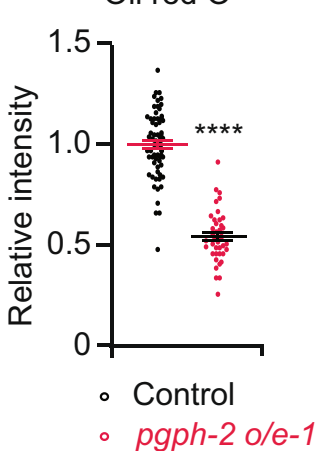

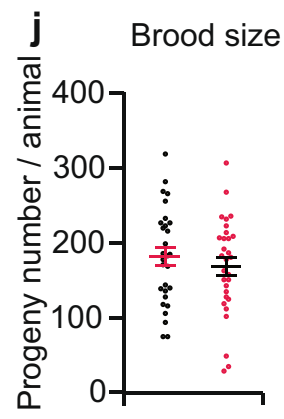

k

Egg laying

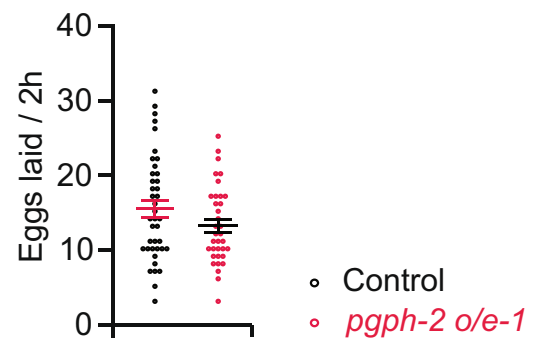

after salt or glucose treatment. In addition, pgph mutant worms are viable and under normal growth conditions do not show any physiological defects, indicating that the accumulation of toxic side-metabolites does not occur to any significant effect in the pgph mutant worm. Other enzymes than PGPH may remove toxic metabolite side products in C. elegans.

We also addressed the role of G3PP in the protection from glucotoxicity and its function related to healthy aging (Figs. 5-7). We show that excess glucose, known to shorten lifespan in
C. elegans ${ }^{14,16,48,58}$, induces PGPH enzymes expression and stimulates glycerol release. Importantly, loss of PGPH exacerbates glucotoxicity, slightly decreases median lifespan and significantly reduces healthspan, as evidenced by a decline in locomotion parameters and the increased susceptibility of worms to various stresses, such as hypoxia, cold and oxidative stresses.

Glucotoxicity in C. elegans has been linked to various metabolic derangements including AMPK inactivation ${ }^{16}$, inhibition of DAF-16 and HSF-1 and downregulation of aquaporin gene 
Fig. 6 pgph-2 overexpression improves healthspan and decreases fat content. a Lifespan of pgph-2 overexpressing animals in comparison to control nematodes. Number of separate lifespan experiments, animals and detailed statistics are shown in Supplementary Data 2. P-value is obtained using the two-sided Mantel-Cox test. b-f Locomotion and body size analyses including the number of body bends per second (b), speed (c), peristaltic speed (d), mean length (e), and mean area (f) in pgph-2 overexpressing animals in comparison to control nematodes at days 3, 5, 7, and 9 of age. Data is shown using violin box plots with denoted median lines from three independent experiments. More than 150 tracks analyzed per condition and exact number of tracks is shown in data source. P-values are obtained by one-way ANOVA with Bonferroni test. $\mathbf{g}$ Violin plots showing pharyngeal pumping rates of control and pgph-2 overexpressing nematodes at days 2, 3, and 4 of age. Sample numbers: $n=35$ (control day 2), $n=27$ ( $p g p h-20 / e 1$ day 2 ), $n=37$ (control day 3 ), $n=30$ (pgph-2 o/e1 day 3), $n=36$ (control day 4), $n=30$ (pgph-2 o/e1 day 4). P-values are obtained by one-way ANOVA with Bonferroni test. h, i Oil red $\mathrm{O}$ staining (h) and quantification (i) in 3 days old adult control and pgph-2 overexpressing nematodes. Scale bars represent $50 \mu \mathrm{m}$. Data represent mean \pm SEM from three independent experiments. Sample numbers: $n=66$ (control), $n=44$ (pgph-2 o/e1). P-value obtained with two-tailed student's $t$ test. j, k Brood size (j) and egg laying (k) in pgph-2 overexpressing animals in comparison to control nematodes. $P$-value obtained with two-tailed student's $t$-test. Data represent mean \pm SEM $n=10$, three independent experiments. Significance in all figures: ${ }^{\star \star \star \star} P<0.0001$. Data are provided as a Source Data file.

expression $^{14}$, glycogen, and fat accumulation ${ }^{48,49}$. We propose that the exacerbation of glucotoxicity by loss of PGPH is due to the accumulation of Gro3P and associated enhanced lipogenesis, excess phospho-glycerolipids, and TG deposition, as well as ROS production through enhanced Gro3P shuttle ${ }^{59}$. In line with these possibilities, decreased Gro3P levels have been reported in longlived mutants ${ }^{60}$.

On the other hand, interventions that prevent damage caused by hyperglycemia and obesity-related disorders, extend lifespan and healthspan in various organisms ${ }^{15-26}$. We now show that the overexpression of $p g p h-2$ protects from glucose-induced toxicity and improves healthspan possibly by preventing lipogenesis and ROS production by the Gro3P shuttle. Fat accumulation in non-adipose tissues causes mitochondrial dysfunction and age-related metabolic decline ${ }^{61}$. Specifically, fat accumulation in ectopic tissues shortens lifespan whereas lipid hydrolysis in $C$. elegans extends lifespan ${ }^{62-66}$. Furthermore, interventions that promote longevity, such as reduced insulin signaling and dietary restriction, are associated with a reduction of ectopic fat in the worm ${ }^{67}$. Our data suggest that by enhancing the conversion of Gro3P to glycerol, $p g p h-2$ overexpression decreases lipogenesis, Gro3P shuttle activity, and ROS production resulting in an extension of healthspan and median lifespan under glucose-rich conditions. Precisely, we show that lipogenesis is increased by the suppression of the PGPH enzymes and reduced by overexpressing $p g p h-2$. Additional work is needed to precisely identify the mechanism(s) whereby variations in G3PP levels modulate healthspan.

One limitation of this study is the minimal information obtained to elaborate on the individual role of pgph-1 in C. elegans biological processes. The high identity in the genetic sequences between the three PGPH enzymes and the close proximity on chromosome $\mathrm{V}$ made it not possible to obtain single pgph-1 mutant animals. Nonetheless, our comparative approach between the double and triple pgph mutant animals as well as $p g p h-2$ and $p g p h-3$ single deletion mutants indicates that $p g p h-2$ plays a major role in glycerol production, hyperosmotic stress response, aging, and glucotoxicity, presumably because of its prominent transcriptional regulation in the intestine, unlike PGPH-3. Moreover, although pgph-1 expression levels are induced by salt and glucose stresses, the fact that the glycerol content, the Gro3P levels, and hyperosmotic stress resistance phenotypes are not different between $p g p h$ double ( $p g p h-2$; $p g p h-3)$ and triple (pgph-1; pgph-2; pgph-3) mutant nematodes indicates that $p g p h-1$ is not as important as $p g p h-2$ for Gro3P phosphatase activity and resistance to hyperosmotic stress.

Calorie restriction is one of the most robust health-promoting interventions yet difficult to sustain ${ }^{15-26}$. Researchers have looked for pharmacological calorie restriction mimetics to promote calorie restriction benefits without the discomfort linked to strict diets to treat cardiometabolic disorders ${ }^{68}$. Interestingly, this work sheds light on a distinct pathway that mimics in part the beneficial effects of calorie restriction without the restriction of calorie intake and changes in fertility. Specifically, this work highlights a significant resemblance in terms of better healthspan parameters and reduced fat accumulation and size, between pgph-2 overexpression and effects of calorie restriction regimens. However, although $p g p h-2$ overexpression significantly increases lifespan under normal conditions and glucotoxic stress, the extension of lifespan is much more prominent with calorie restriction or in insulin-like signaling pathway mutants in C. elegans ${ }^{20,69}$.

In conclusion, the results show marked effects of variations in PGPH/G3PP levels in terms of healthspan but modest effects on lifespan in C. elegans. However, it should be pointed out that improved healthspan does not need to correlate with large increases in lifespan, and the large lifespan increase in some $C$. elegans mutants does not scale up to mammals or primates at all, but the healthspan does well. Hence, it may be hypothesized that G3PP activation might provide an entirely novel approach to prevent and treat diseases due to nutritional excess such as obesity, diabetes, NASH, and CVD. In fact, a recent study has identified G3PP as a new potential candidate longevity gene via an exome-wide association study in a long-lived centenarian cohort in comparison to younger individuals ${ }^{70}$. Thus, in the worm, enhanced G3PP activity protects from fat deposition and promotes healthy aging, and aging in normal and excess glucose conditions, which is by far the most single contributor to cardiometabolic disorders.

\section{Methods}

C. elegans strains, maintenance, and RNAi interference. Nematodes were maintained and synchronized using standard culture methods ${ }^{71}$. We used the following strains: N2 Bristol, pgph-2p::GFP (rCesF44E7.2::GFP + pCeh361), pgph3p::GFP (rCesC53A3.2::GFP + pCeh361) that were obtained from the Caenorhabditis Genetics Center. pgph-3(tm3391) was provided by Dr. Mitani (Nation bio-resource project of the Mext, Japan). pgph-2(xq830-1), pgph-2(xq830-2), pgph2(xq830); pgph-3(tm3391) and pgph-1(xq829); pgph-2(xq830); pgph-3(tm3391) strains were generated in our lab using CRISPR-CAS9 technology and will be available upon request. Two RNAi clones were obtained from the Dharmacon library and RNAi feeding experiments were performed as described in ${ }^{72}$, and bacteria transformed with empty vector were used as control. For all RNAi experiments, phenotypes were scored with the F1 generation.

Synchronization methods. Two synchronization methods were used in this study For experiments that require a small number of progeny including lifespan, locomotion, glucotoxicity, stress resistance, brood size, pharyngeal pumping, and swimming behavior experiments, animals were synchronized by transferring 5-8 gravid hermaphrodites to fresh agar plates to permit egg laying for four hours, then the hermaphrodites were removed and eggs were allowed to hatch and grow until they reached the experimental stage. For biochemical experiments including RNA expression, glycerol, and metabolite measurement experiments, the nematodes were synchronized by the standard hypochlorite bleaching $\operatorname{method}^{73}$. 
Control

pgph-2 o/e-1

a
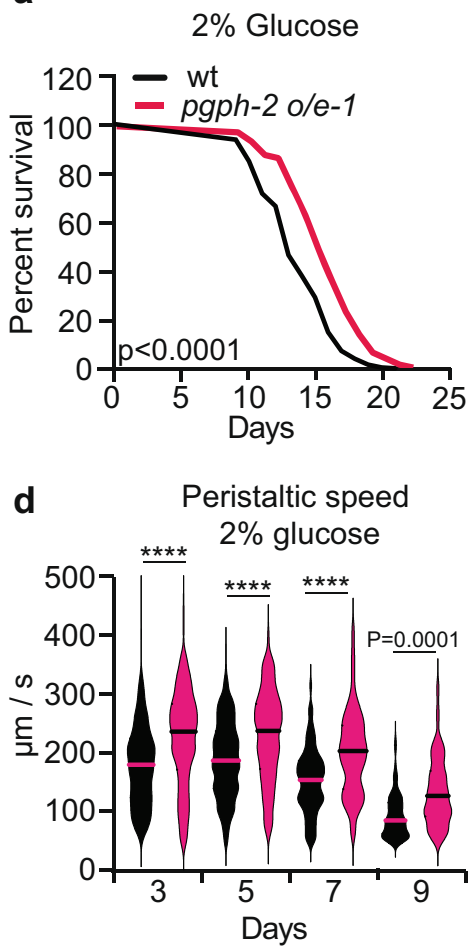

b
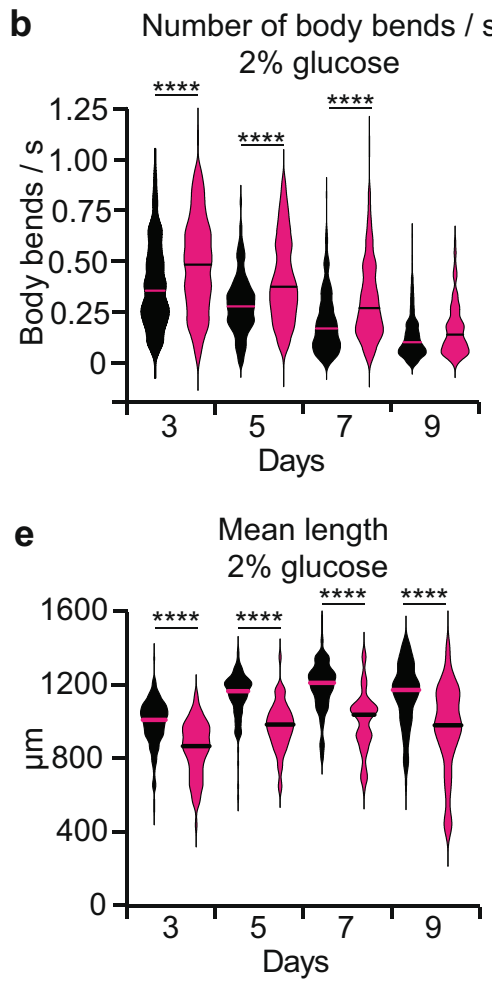

C

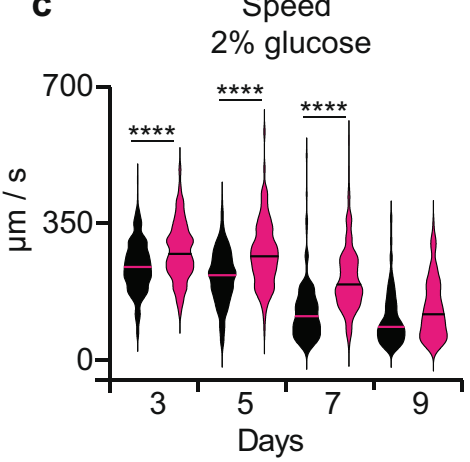

$\mathbf{f}$

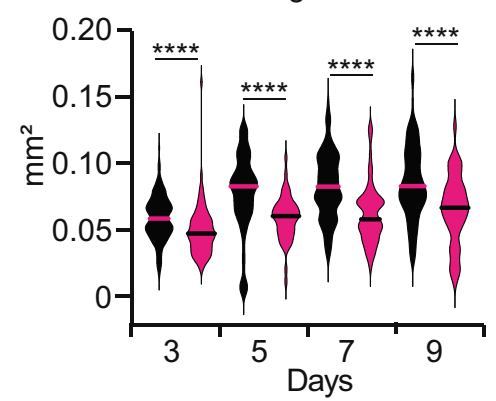

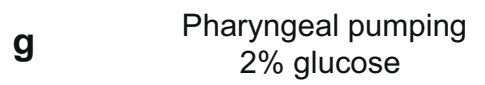
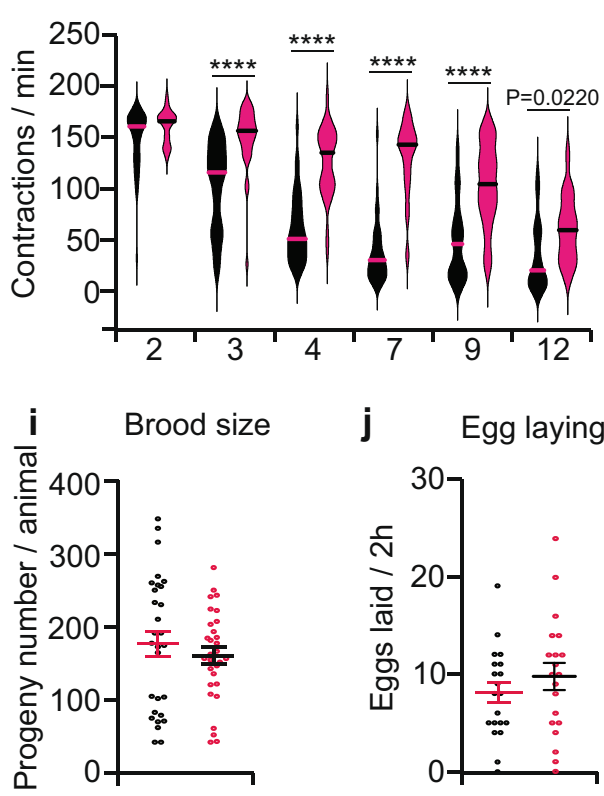

j Egg laying

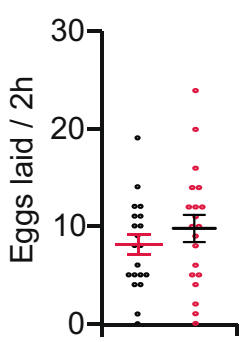

- Control - gluc

- pgph-2 o/e-1 - gluc

h
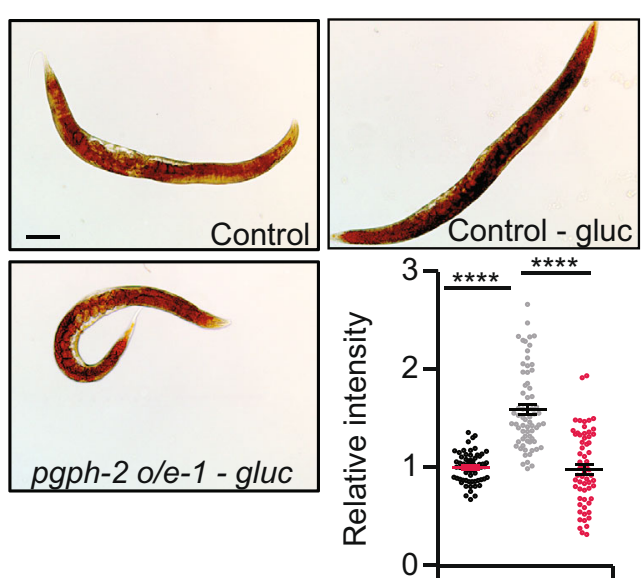

- Control

- Control - gluc - pgph-2 o/e-1 - gluc
Plasmid construction and overexpressing transgenic strains. The overexpressing $p g p h-2$ and $p g p h-3$ plasmids were generated using Clontech In-Fusion PCR Cloning Kit according to manufacturer's protocol ${ }^{74}$. The promoter region and genes were amplified from N2 worm genomic DNA and were cloned into ppD49.26 vector using $s b f-1$ and $k p n-1$ cloning sites for $p g p h-2$ and $s b f-1$ and EcoR-V for $p g p h-3$. Cloning mixtures were transformed using stellar competent cells according to the manufacturer's protocol. $10 \mathrm{ng} / \mathrm{ul}$ of plasmid DNA, $20 \mathrm{ng} / \mathrm{ul} \mathrm{mCherry,} \mathrm{and} 170 \mathrm{ng} / \mathrm{ul}$
pBluescript were injected into worm gonad arms of WT worms and the positive lines were maintained after transmission to the $\mathrm{F} 3$ generation.

Transcription factor RNAi screen. Briefly, 3-5 adult worms were grown on RNA plates and allowed to lay eggs. After 3 days, the plates were scored for reduced GFP fluorescence signal at basal conditions using a fluorescence dissecting microscope. Of 
Fig. 7 pgph-2 overexpression increases lifespan under excess glucose and reduces glucose-induced fat deposition. a Survival curves of pgph-2 overexpressing animals in comparison to control nematodes in excess glucose conditions. Number of separate experiments, animals, and detailed statistics are shown in Supplementary Data 3. P-values are obtained by the two-sided Mantel-Cox test. b-f Locomotion and body size analyses including the number of body bends per second (b), speed (c), peristaltic speed (d), mean length (e), and mean area (f) in pgph-2 overexpressing animals in comparison to control nematodes grown on plates supplemented with $2 \%$ glucose at days 3, 5, 7, and 9 of age. Data is represented using violin box plots with denoted median lines from three independent experiments. More than 150 tracks were analyzed per condition and an exact number of tracks is shown in the data source. $\mathbf{g}$ Violin box plots showing pharyngeal pumping rates of control and pgph-2 overexpressing nematodes grown on plates supplemented with $2 \%$ glucose at the indicated age. Sample numbers: $n=36$ (control day 2), $n=30$ (pgph-2 o/e1 day 2), $n=34$ (control days 3, 4, 7, 9, 12), $n=30$ (pgph-2 o/e1 days $3,4,7,9,12)$. $\mathbf{h}$ Oil red $O$ staining and quantification in 3 days old adult control and pgph-2 overexpressing nematodes grown on plates supplemented with $2 \%$ glucose. Scale bars represent $50 \mu \mathrm{m}$. Data represent mean \pm SEM from three independent experiments. Sample numbers: $n=65$ (control), $n=68$ (control-2\% glucose), $n=63$ (pgph-2 o/e1). i, j Brood size (i) and egg laying (j) in indicated pgph-2 overexpressing animals grown on plates supplemented with $2 \%$ glucose in comparison to control nematodes. Data represent mean $\pm S E M, n=10$, three independent experiments. Significance in all figures: ${ }_{\star \star \star \star \star} P<0.0001$. From $\mathbf{b}-\mathbf{j}, P$-values are obtained by one-way ANOVA with the Bonferroni test. Data are provided as a Source Data file.

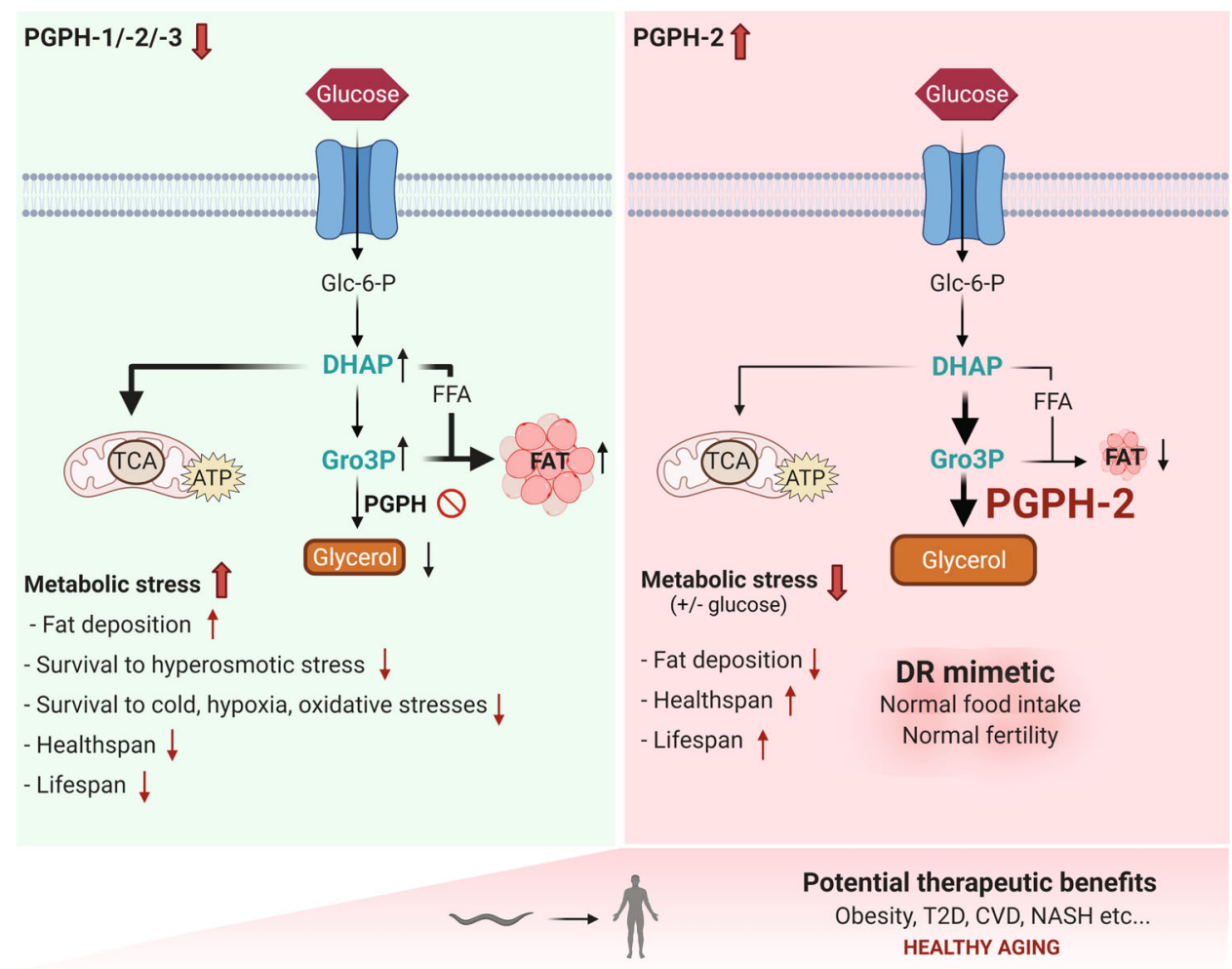

Fig. 8 Graphical abstract summarizing the main findings. We have characterized the function of the PGPH enzymes in C. elegans. PGPH enzymes act as glycerol-3-phosphate phosphatase (G3PP) and are responsible for glycerol production via the direct hydrolysis of glycerol-3-phosphate. Loss of PGPH enzymes decreases resistance to diverse stresses, shortens lifespan and healthspan and exacerbates glucotoxicity. In contrast, the overexpression of PGPH-2, mimics calorie restriction without changes in food intake and fertility, decreases fat deposition, protects from glucose-induced toxicity and promotes healthy aging. Overall, the results identify G3PP as a candidate therapeutic avenue to treat obesity and age-related cardiometabolic disorders, and to promote healthy aging.

their progeny, around 15 animals were handpicked and placed on corresponding RNAi plates supplemented with $400 \mathrm{mM} \mathrm{NaCl}$ for two hours. The plates were screened for reduced GFP signal and a secondary screen to confirm positive hits followed.

\footnotetext{
Lifespan and glucose toxicity assays. All lifespan and glucose toxicity curves were performed at $20^{\circ} \mathrm{C}$. Briefly, C. elegans nematodes were synchronized as described above. For glucose toxicity, the animals were constantly transferred to $E$. coli seeded agar plates supplemented with $2 \%$ glucose. Three days after egg preparation, around 120 animals were manually transferred (40 animals per plate in triplicates) to fresh plates. Worms were transferred daily for the first week and every other day afterwards until the aged population is well separated from growing larvae. Worms were scored daily and were considered alive if they responded to gentle tapping with a platinum wire. Worms that crawled off the plate or died from bagging or internal hatching were censored from the analysis.
}

Locomotion assays. Along with the lifespan and glucose toxicity assays, the locomotion behaviors of the nematodes were filmed for $30 \mathrm{~s}$ at days $3,5,7$, and 9 using the Wormtracker system (MBF Bioscience). More than 200 tracks per genotype/condition were generated in three independent repeats. Movies were analyzed and average speed, peristaltic speed, number of body bends per second were computed using software Wormlab.

Paraquat resistance assay. Resistance to $100 \mathrm{mM}$ paraquat was performed as previously described ${ }^{75}$. Briefly, 4-8 synchronized L4-young adult animals were transferred to the wells of a 96 well plates containing $100 \mathrm{mM}$ paraquat dissolved in M9 solution. We monitored at least 12 wells per condition.

Hyperosmotic stress resistance assay. Hyperosmotic stress resistance was assayed as previously described ${ }^{33}$. Briefly, synchronized 1-day adult worms were transferred to $400 \mathrm{mM} \mathrm{NaCl}$ plates. Worms that responded by the movement to touch with the platinum wire were considered alive. Survival was measured daily.

Hypoxia stress. One-day-old adult animals were transferred to corresponding RNAi plates and were kept in a Bio-Bag Environmental Chamber Type A (Becton 
Dickinson Microbiology Systems) for $24 \mathrm{~h}$ at $20^{\circ} \mathrm{C}$. The number of crawling animals was scored at indicated time points and recovery rates were calculated.

Cold stress. For cold stress, 1-day adult animals were transferred to corresponding RNAi plates and were kept at $4{ }^{\circ} \mathrm{C}$ for $16 \mathrm{~h}$. The plates were transferred back to $20^{\circ} \mathrm{C}$ and the number of crawling animals was scored at indicated time points to calculate recovery rates.

RNA extraction and RT-PCR. Synchronized young adult nematodes were harvested and total RNA was extracted with Trizol. Reverse transcription and qRTPCRs were performed ${ }^{3}$. Transcripts were normalized to $c d c 42$. Primers sequences are available in Supplementary Data 8.

Oil red $\mathrm{O}$ staining. Oil red $\mathrm{O}$ staining was performed as previously described with small modifications ${ }^{76}$. Briefly, synchronized young adult animals were collected with M9 buffer and washed twice with PBS1x-0.01\% triton X. Animal pellets were treated for $15 \mathrm{~min}$ with PBS1x, $0.01 \%$ Triton X, 60\% isopropanol solution and transferred to Eppendorf tubes and incubated in $60 \%$ oil red $\mathrm{O}$ solution overnight. The oil red $\mathrm{O}$ stock solution was equilibrated for $>3$ days and the $60 \%$ diluted solution was equilibrated overnight and filtered two times on the day of the experiment. Images were taken using a Leica microscope and were analyzed using Image J.

Metabolite extraction and glycerol determination. $\mathrm{NaCl}$ treatment: Synchronized L4/young adult animals exposed or not to $400 \mathrm{mM} \mathrm{NaCl}$ for $2 \mathrm{~h}$ and were harvested and washed with M9 buffer three times adjusted to match plate salinity. Pellets were flash frozen in liquid N2.

Glucose treatment: Synchronized L1 animals by sodium hypochlorite were plated on NGM plates or plates supplemented with $2 \%$ glucose for $48 \mathrm{~h}$ then harvested with M9 buffer and washed three times before flash freezing in liquid N2.

Glucose-NaCl treatment: Synchronized L1 animals by sodium hypochlorite were plated on NGM plates or plates supplemented with $2 \%$ glucose and grown for $48 \mathrm{~h}$ at $20^{\circ} \mathrm{C}$. The animals were then collected with M9 buffer with adjusted salinities and exposed to plates supplemented with $400 \mathrm{mM} \mathrm{NaCl}$ for $3 \mathrm{~h}$. Animals were harvested with M9 buffer and washed three times before flash freezing in liquid N2.

Glycerol levels were determined using a radiometric glycerol assay using $\left[\gamma^{-32} \mathrm{P}\right]$ ATP and glycerol kinase and were normalized to protein content measured by $\mathrm{BCA}^{31}$.

Metabolites were analyzed by LC-MS/MS ${ }^{3}$ with small modifications. Worm pellets $(\sim 200 \mathrm{~mL})$ were collected in CK14-2 $\mathrm{mL}$ lysing kit tubes (Bertin Technologies), flash frozen, and stored at $-80^{\circ} \mathrm{C}$. Samples were slowly thawed and homogenized using 4.25 volumes of ice-cold aqueous methanol $98.8 \%, 2.4 \mathrm{mM}$ ammonium acetate $(\mathrm{pH} 9)$, containing $10 \mathrm{mM}\left({ }^{13} \mathrm{C}_{10},{ }^{15} \mathrm{~N}_{5}\right)$-AMP as internal standard, using bead beating (Precellys plus Cryolys cooler, Bertin Technologies protocol: $6000 \mathrm{rpm}, 2 \times 25 \mathrm{~s}, 15 \mathrm{~s}$ pause) and $2 \mathrm{~min}$ sonication (cycles of $10 \mathrm{sec} \mathrm{on} /$ off, output of $150 \mathrm{~W}$ ) in a cup-horn sonicator filled with water and ice. Homogenates were incubated $15 \mathrm{~min}$ on ice and centrifuged $20,000 \mathrm{~g}, 15 \mathrm{~min}$ at $4{ }^{\circ} \mathrm{C}$. Water-soluble metabolites were then extracted from the supernatant by liquid-liquid extraction and analyzed as described ${ }^{3}$. Relative amounts were normalized to protein content using BCA.

Triglyceride determination. Synchronized 1-day adult animals were harvested and washed with M9 buffer and pellets were flash frozen in liquid $\mathrm{N}_{2}$. Pellets were then crushed in mortar and pestle on dry ice and worm powder was resuspended in $300 \mu \mathrm{l}$ of $5 \%$ NP40. Extracts were then collected in tubes, sonicated, then heated at $100{ }^{\circ} \mathrm{C}$ for $5 \mathrm{~min}$, and centrifuged at $15,994 \times \mathrm{g}, 4^{\circ} \mathrm{C}$, for $5 \mathrm{~min}$. Supernatants were used for TG measurements using TG determination kit from Sigma Aldrich according to the manufacturer's protocol and pellets were resuspended in $0.2 \mathrm{~N}$ $\mathrm{NaOH}$ and protein amounts were determined using BCA.

Pharyngeal pumping. Pharyngeal pumping rates were measured by counting the number of grinder movements per $30 \mathrm{~s}$ using a stereomicroscope. The worms were always on a lawn of food at rest. Pharyngeal pumping rates were measured in at least ten worms per condition.

Brood size. Single L4 animals were transferred to fresh agar plates seeded with $E$. Coli OP50 bacteria. The animals were transferred individually to fresh plates every day until they stopped laying eggs after 5-6 days. The progeny number was counted 2 days after egg laying and the brood size was determined by the sum of the number of progeny produced by an individual hermaphrodite.

Egg laying. Single synchronized 1-day adult animals were transferred to fresh OP50-seeded NGM plates and left for $2 \mathrm{~h}$. Animals were removed from the plates and the number of eggs was counted. We used ten replicates per strain and the experiment was repeated three times.
Swimming exercise. We used the WMicroTracker machine (Phylum Tech) ${ }^{77}$ to track the swimming activity of the strains. Day 1 adult animals grown on NGM were transferred to 96 well plates containing $100 \mu \mathrm{l}$ of M9 buffer with 30 animals per well in triplicates, and the swimming behavior was tracked and computed for $10 \mathrm{~h}$.

Transcriptional reporters imaging and analysis. Transcriptional reporter strains $p g p h-2 p:: G F P$ and $p g p h-3 p:: G F P$ were obtained from the Dr. Ballie's library (CGC). Animals were immobilized in $5 \mathrm{mM}$ levamisole and mounted on $2 \%$ agarose pads and imaged using the Leica SP5 confocal microscope.

Statistical analyses. Data are expressed as means \pm SEM. Statistical analyses were performed by student's $t$-test for two groups or one-way ANOVA for multiple groups using Graphpad. For survival curves, we used the Log-rank Mantel-Cox test. Significance is indicated in the figures and legends or included in the supplementary Data.

Reporting summary. Further information on research design is available in the Nature Research Reporting Summary linked to this article.

\section{Data availability}

All relevant data generated or analyzed during this study are included in this manuscript and/or its supplementary information. Metabolomics datasets have been deposited at Metabolights ${ }^{78}$ and are available under accession code MTBLS3485. The data underlying Figs. 1-7 and Supplementary Figs. 1-11 are provided as Source data. Any remaining raw data will be available from the corresponding author upon reasonable request. Source data are provided with this paper.

Received: 12 January 2021; Accepted: 7 December 2021; Published online: 11 January 2022

\section{References}

1. Prentki, M. \& Madiraju, S. R. Glycerolipid metabolism and signaling in health and disease. Endocr. Rev. 29, 647-676 (2008).

2. Prentki, M. \& Madiraju, S. R. Glycerolipid/free fatty acid cycle and islet betacell function in health, obesity and diabetes. Mol. Cell Endocrinol. 353, 88-100 (2012)

3. Mugabo, Y. et al. Identification of a mammalian glycerol-3-phosphate phosphatase: role in metabolism and signaling in pancreatic beta-cells and hepatocytes. Proc. Natl Acad. Sci. USA 113, E430-E439 (2016).

4. Rose, Z. B. Phosphoglycolate phosphatase from human red blood cells. Arch Biochem Biophys. 208, 602-609 (1981).

5. Possik, E., Madiraju, S. R. M. \& Prentki, M. Glycerol-3-phosphate phosphatase/ PGP: role in intermediary metabolism and target for cardiometabolic diseases. Biochimie 143, 18-28 (2017).

6. Kuznetsova, E. et al. Functional diversity of haloacid dehalogenase superfamily phosphatases from Saccharomyces cerevisiae: biochemical, structural, and evolutionary insights. J. Biol. Chem. 290, 18678-18698 (2015).

7. Segerer, G. et al. An essential developmental function for murine phosphoglycolate phosphatase in safeguarding cell proliferation. Sci. Rep. 6, 35160 (2016).

8. Collard, F. et al. A conserved phosphatase destroys toxic glycolytic side products in mammals and yeast. Nat. Chem. Biol. 12, 601-607 (2016).

9. Seifried, A. et al. Evolutionary and structural analyses of mammalian haloacid dehalogenase-type phosphatases AUM and chronophin provide insight into the basis of their different substrate specificities. J. Biol. Chem. 289, 3416-3431 (2014).

10. Aston, L. M. Glycaemic index and metabolic disease risk. Proc. Nutr. Soc. 65 125-134 (2006)

11. Venn, B. J. \& Green, T. J. Glycemic index and glycemic load: measurement issues and their effect on diet-disease relationships. Eur. J. Clin. Nutr. 61, S122-S131 (2007).

12. Mlekusch, W., Lamprecht, M., Ottl, K., Tillian, M. \& Reibnegger, G. A glucose-rich diet shortens longevity of mice. Mech. Ageing Dev. 92, 43-51 (1996).

13. Ruff, J. S. et al. Human-relevant levels of added sugar consumption increase female mortality and lower male fitness in mice. Nat. Commun. 4, 2245 (2013).

14. Lee, S. J., Murphy, C. T. \& Kenyon, C. Glucose shortens the life span of C. elegans by downregulating DAF-16/FOXO activity and aquaporin gene expression. Cell Metab. 10, 379-391 (2009).

15. Smith, E. D. et al. Age- and calorie-independent life span extension from dietary restriction by bacterial deprivation in Caenorhabditis elegans. BMC Dev. Biol. 8, 49 (2008). 
16. Schulz, T. J. et al. Glucose restriction extends Caenorhabditis elegans life span by inducing mitochondrial respiration and increasing oxidative stress. Cell Metab. 6, 280-293 (2007).

17. Lee, G. D. et al. Dietary deprivation extends lifespan in Caenorhabditis elegans. Aging Cell 5, 515-524 (2006).

18. Wu, Z. et al. Dietary restriction extends lifespan through metabolic regulation of innate immunity. Cell Metab. 29, 1192-1205 e8 (2019).

19. Matai, L. et al. Dietary restriction improves proteostasis and increases life span through endoplasmic reticulum hormesis. Proc. Natl Acad. Sci. USA 116, 17383-17392 (2019).

20. Mair, W. \& Dillin, A. Aging and survival: the genetics of life span extension by dietary restriction. Annu Rev. Biochem 77, 727-754 (2008).

21. Lin, S. J. et al. Calorie restriction extends Saccharomyces cerevisiae lifespan by increasing respiration. Nature 418, 344-348 (2002).

22. Partridge, L., Piper, M. D. \& Mair, W. Dietary restriction in Drosophila. Mech. Ageing Dev. 126, 938-950 (2005).

23. Colman, R. J. et al. Caloric restriction delays disease onset and mortality in rhesus monkeys. Science 325, 201-204 (2009).

24. Mattison, J. A. et al. Impact of caloric restriction on health and survival in rhesus monkeys from the NIA study. Nature 489, 318-321 (2012).

25. Fontana, L., Partridge, L. \& Longo, V. D. Extending healthy life span-from yeast to humans. Science 328, 321-326 (2010).

26. Fontana, L. \& Partridge, L. Promoting health and longevity through diet: from model organisms to humans. Cell 161, 106-118 (2015).

27. Gaudet, D. et al. Glycerol as a correlate of impaired glucose tolerance: dissection of a complex system by use of a simple genetic trait. Am. J. Hum. Genet 66, 1558-1568 (2000).

28. Prentki, M., Peyot, M. L., Masiello, P. \& Madiraju, S. R. M. Nutrient-induced metabolic stress, adaptation, detoxification, and toxicity in the pancreatic betacell. Diabetes 69, 279-290 (2020).

29. Burg, M. B. \& Ferraris, J. D. Intracellular organic osmolytes: function and regulation. J. Biol. Chem. 283, 7309-7313 (2008).

30. Yancey, P. H. Organic osmolytes as compatible, metabolic and counteracting cytoprotectants in high osmolarity and other stresses. J. Exp. Biol. 208, 2819-2830 (2005).

31. Lamitina, S. T., Morrison, R., Moeckel, G. W. \& Strange, K. Adaptation of the nematode Caenorhabditis elegans to extreme osmotic stress. Am. J. Physiol. Cell Physiol. 286, C785-C791 (2004).

32. Rohlfing, A. K., Miteva, Y., Hannenhalli, S. \& Lamitina, T. Genetic and physiological activation of osmosensitive gene expression mimics transcriptional signatures of pathogen infection in C. elegans. PLoS One 5, e9010 (2010)

33. Possik, E. et al. FLCN and AMPK confer resistance to Hyperosmotic stress via remodeling of glycogen stores. PLoS Genet 11, e1005520 (2015).

34. Possik, E. \& Pause, A. Glycogen: a must have storage to survive stressful emergencies. Worm 5, e1156831 (2016).

35. Frazier, H. N. 3rd \& Roth, M. B. Adaptive sugar provisioning controls survival of C. elegans embryos in adverse environments. Curr. Biol. 19, 859-863 (2009).

36. Burkewitz, K., Choe, K. P., Lee, E. C., Deonarine, A. \& Strange, K. Characterization of the proteostasis roles of glycerol accumulation, protein degradation and protein synthesis during osmotic stress in C. elegans. PLoS One 7, e34153 (2012).

37. Lamitina, T., Huang, C. G. \& Strange, K. Genome-wide RNAi screening identifies protein damage as a regulator of osmoprotective gene expression. Proc. Natl Acad. Sci. USA 103, 12173-12178 (2006).

38. O'Rourke, S. M., Herskowitz, I. \& O'Shea, E. K. Yeast go the whole HOG for the hyperosmotic response. Trends Genet 18, 405-412 (2002).

39. Scolaro, G. et al. Increased expression of pgph-1, T23F2.4, and cyp-14A5 in C. elegans dpy-7 mutants and by high salt. MicroPubl. Biol. https://doi.org/ 10.17912/micropub.biology.000136 (2019).

40. Glenn, C. F. et al. Behavioral deficits during early stages of aging in Caenorhabditis elegans result from locomotory deficits possibly linked to muscle frailty. J. Gerontol. A Biol. Sci. Med Sci. 59, 1251-1260 (2004).

41. Wakabayashi, T., Kitagawa, I. \& Shingai, R. Neurons regulating the duration of forward locomotion in Caenorhabditis elegans. Neurosci. Res 50, 103-111 (2004).

42. Herndon, L. A. et al. Stochastic and genetic factors influence tissue-specific decline in ageing C. elegans. Nature 419, 808-814 (2002).

43. Rollins, J. A., Howard, A. C., Dobbins, S. K., Washburn, E. H. \& Rogers, A. N. Assessing health span in Caenorhabditis elegans: lessons from short-lived mutants. J. Gerontol. A Biol. Sci. Med Sci. 72, 473-480 (2017).

44. Huang, C., Xiong, C. \& Kornfeld, K. Measurements of age-related changes of physiological processes that predict lifespan of Caenorhabditis elegans. Proc. Natl Acad. Sci. USA 101, 8084-8089 (2004).

45. Seo, Y., Kingsley, S., Walker, G., Mondoux, M. A. \& Tissenbaum, H. A Metabolic shift from glycogen to trehalose promotes lifespan and healthspan in Caenorhabditis elegans. Proc. Natl Acad. Sci. USA 115, E2791-E2800 (2018).

46. Bansal, A., Zhu, L. J., Yen, K. \& Tissenbaum, H. A. Uncoupling lifespan and healthspan in Caenorhabditis elegans longevity mutants. Proc. Natl Acad. Sci. USA 112, E277-E286 (2015).

47. Newell Stamper, B. L. et al. Movement decline across lifespan of Caenorhabditis elegans mutants in the insulin/insulin-like signaling pathway. Aging Cell 17, e12704 (2018).

48. Lee, D. et al. SREBP and MDT-15 protect C. elegans from glucose-induced accelerated aging by preventing accumulation of saturated fat. Genes Dev. 29, 2490-2503 (2015).

49. Gusarov, I. et al. Glycogen controls Caenorhabditis elegans lifespan and resistance to oxidative stress. Nat. Commun. 8, 15868 (2017).

50. Alcantar-Fernandez, J., Navarro, R. E., Salazar-Martinez, A. M., PerezAndrade, M. E. \& Miranda-Rios, J. Caenorhabditis elegans respond to highglucose diets through a network of stress-responsive transcription factors. PLoS One 13, e0199888 (2018).

51. Lopez-Otin, C., Galluzzi, L., Freije, J. M. P., Madeo, F. \& Kroemer, G. Metabolic control of longevity. Cell 166, 802-821 (2016).

52. Fontana, L. \& Hu, F. B. Optimal body weight for health and longevity: bridging basic, clinical, and population research. Aging Cell 13, 391-400 (2014).

53. Ewald, C. Y., Castillo-Quan, J. I. \& Blackwell, T. K. Untangling longevity, dauer, and healthspan in Caenorhabditis elegans insulin/IGF-1-signalling. Gerontology 64, 96-104 (2018).

54. Hansen, M. \& Kennedy, B. K. Does longer lifespan mean longer healthspan? Trends Cell Biol. 26, 565-568 (2016).

55. Bar, D. Z. et al. Cell size and fat content of dietary-restricted Caenorhabditis elegans are regulated by ATX-2, an mTOR repressor. Proc. Natl Acad. Sci. USA 113, E4620-E4629 (2016).

56. Gerin, I. et al. Phosphoglycolate has profound metabolic effects but most likely no role in a metabolic DNA response in cancer cell lines. Biochem J. 476, 629-643 (2019).

57. Liberti, M. V. \& Locasale, J. W. Metabolism: a new layer of glycolysis. Nat. Chem. Biol. 12, 577-578 (2016).

58. Schlotterer, A. et al. C. elegans as model for the study of high glucosemediated life span reduction. Diabetes 58, 2450-2456 (2009).

59. Mracek, T. et al. ROS generation and multiple forms of mammalian mitochondrial glycerol-3-phosphate dehydrogenase. Biochim Biophys. Acta 1837, 98-111 (2014).

60. Gao, A. W. et al. Identification of key pathways and metabolic fingerprints of longevity in C. elegans. Exp. Gerontol. 113, 128-140 (2018).

61. Petersen, K. F. et al. Mitochondrial dysfunction in the elderly: possible role in insulin resistance. Science 300, 1140-1142 (2003).

62. Schmeisser, S. et al. Muscle-Specific lipid hydrolysis prolongs lifespan through global lipidomic remodeling. Cell Rep. 29, 4540-4552 e8 (2019).

63. Zaarur, N. et al. ATGL-1 mediates the effect of dietary restriction and the insulin/IGF-1 signaling pathway on longevity in C. elegans. Mol. Metab. 27, 75-82 (2019).

64. Johnson, A. A. \& Stolzing, A. The role of lipid metabolism in aging, lifespan regulation, and age-related disease. Aging Cell 18, el3048 (2019).

65. Lapierre, L. R., Melendez, A. \& Hansen, M. Autophagy links lipid metabolism to longevity in C. elegans. Autophagy 8, 144-146 (2012).

66. Wang, M. C., O’Rourke, E. J. \& Ruvkun, G. Fat metabolism links germline stem cells and longevity in C. elegans. Science 322, 957-960 (2008).

67. Palikaras, K. et al. Ectopic fat deposition contributes to age-associated pathology in Caenorhabditis elegans. J. Lipid Res 58, 72-80 (2017).

68. Madeo, F., Carmona-Gutierrez, D., Hofer, S. J. \& Kroemer, G. Caloric restriction mimetics against age-associated disease: targets, mechanisms, and therapeutic potential. Cell Metab. 29, 592-610 (2019).

69. Lapierre, L. R. \& Hansen, M. Lessons from C. elegans: signaling pathways for longevity. Trends Endocrinol. Metab. 23, 637-644 (2012).

70. Torres, G. G. et al. Exome-wide association study identifies FN3KRP and PGP as new candidate longevity genes. J. Gerontol. A Biol. Sci. Med Sci. 76, 786-795 (2021).

71. Brenner, S. The genetics of Caenorhabditis elegans. Genetics 77, 71-94 (1974)

72. Kamath, R. S., Martinez-Campos, M., Zipperlen, P., Fraser, A. G. \& Ahringer, J. Effectiveness of specific RNA-mediated interference through ingested double-stranded RNA in Caenorhabditis elegans. Genome Biol. 2, RESEARCH0002 (2001).

73. Porta-de-la-Riva, M., Fontrodona, L., Villanueva, A. \& Ceron, J. Basic Caenorhabditis elegans methods: synchronization and observation. J. Vis. Exp. 10, e4019 (2012).

74. Zhu, B., Cai, G., Hall, E. O. \& Freeman, G. J. In-fusion assembly: seamless engineering of multidomain fusion proteins, modular vectors, and mutations. Biotechniques 43, 354-359 (2007).

75. Possik, E. \& Pause, A. Measuring oxidative stress resistance of Caenorhabditis elegans in 96-well microtiter plates. J. Vis. Exp. 9, e52746 (2015). 
76. Settembre, C. et al. TFEB controls cellular lipid metabolism through a starvation-induced autoregulatory loop. Nat. Cell Biol. 15, 647-658 (2013).

77. Simonetta, S. H. \& Golombek, D. A. An automated tracking system for Caenorhabditis elegans locomotor behavior and circadian studies application. J. Neurosci. Methods 161, 273-280 (2007).

78. Haug, K. et al. MetaboLights: a resource evolving in response to the needs of its scientific community. Nucleic Acids Res 48, D440-D444 (2020).

\section{Acknowledgements}

This study was supported by funds from the Canadian Institutes of Health Research (to M.P. and S.R.M.M.). M.P. holds the Canada Research Chair in Diabetes and Metabolism. E.P. is a recipient of postdoctoral fellowships from Diabetes Canada. We thank the Metabolomics and Imaging facilities of CRCHUM. We acknowledge the Caenorhabditis Genetic Center and the Mitani Lab for C. elegans strains. We also gratefully acknowledge Dr. Siegfried Hekimi and Dr. Richard Roy (McGill University) for helpful discussions. We also thank Dr. Parker's lab members, specifically Audrey Labarre, Gilles Tossing, Yasmin Fard Ghassemi, and James Doyle for help and support.

\section{Author contributions}

E.P., J.A.P., S.R.M.M., and M.P. contributed to the research design. E.P., C.S., A.A., J.M., H.E., W.K., Y.B, L.C., and A.O., contributed to experiment performances. E.P., S.R.M.M., J.A.P., and M.P. contributed to the analysis of the data, and E.P., S.R.M.M., and M.P. contributed to the writing of the paper.

\section{Competing interests}

The authors declare no competing interests.

\section{Additional information}

Supplementary information The online version contains supplementary material available at https://doi.org/10.1038/s41467-021-27803-6.

Correspondence and requests for materials should be addressed to S. R. Murthy Madiraju or Marc Prentki.

Peer review information Nature Communications thanks Pingsheng Liu and the other, anonymous, reviewer(s) for their contribution to the peer review of this work.

Reprints and permission information is available at http://www.nature.com/reprints

Publisher's note Springer Nature remains neutral with regard to jurisdictional claims in published maps and institutional affiliations.

cc (i) Open Access This article is licensed under a Creative Commons Attribution 4.0 International License, which permits use, sharing, adaptation, distribution and reproduction in any medium or format, as long as you give appropriate credit to the original author(s) and the source, provide a link to the Creative Commons license, and indicate if changes were made. The images or other third party material in this article are included in the article's Creative Commons license, unless indicated otherwise in a credit line to the material. If material is not included in the article's Creative Commons license and your intended use is not permitted by statutory regulation or exceeds the permitted use, you will need to obtain permission directly from the copyright holder. To view a copy of this license, visit http://creativecommons.org/ licenses/by/4.0/.

(C) The Author(s) 2022 